\title{
Pressure-driven flow of suspensions: simulation and theory
}

\author{
By PRABHU R. NOTT $\dagger$ AND JOHN F. BRADY \\ Division of Chemistry and Chemical Engineering, California Institute of Technology, \\ Pasadena, CA 91125, USA
}

(Received 27 December 1992 and in revised form 3 April 1994)

Dynamic simulations of the pressure-driven flow in a channel of a non-Brownian suspension at zero Reynolds number were conducted using Stokesian Dynamics. The simulations are for a monolayer of identical particles as a function of the dimensionless channel width and the bulk particle concentration. Starting from a homogeneous dispersion, the particles gradually migrate towards the centre of the channel, resulting in an homogeneous concentration profile and a blunting of the particle velocity profile. The time for achieving steady state scales as $(H / a)^{3} a /\langle u\rangle$, where $H$ is the channel width, $a$ the radii of the particles, and $\langle u\rangle$ the average suspension velocity in the channel. The concentration and velocity profiles determined from the simulations are in qualitative agreement with experiment.

A model for suspension flow has been proposed in which macroscopic mass, momentum and energy balances are constructed and solved simultaneously. It is shown that the requirement that the suspension pressure be constant in directions perpendicular to the mean motion leads to particle migration and concentration variations in inhomogeneous flow. The concept of the suspension 'temperature' - a measure of the particle velocity fluctuations - is introduced in order to provide a nonlocal description of suspension behaviour. The results of this model for channel flow are in good agreement with the simulations.

\section{Introduction}

The behaviour of flowing suspensions has been a matter of interest for many years and there has recently been a considerable increase in research activity. From a practical point of view, the importance of this subject stems from the many applications in industry where the processing and transport of suspensions is an important operation. Of equal importance is the need for a fundamental understanding of the physical phenomena occurring in suspensions. Though there is as yet no complete theory that fully accounts for all the interactions and resultant phenomena in concentrated suspensions, recent advances in experimental and analytical techniques have led to some progress in the understanding of these systems.

The present work focuses on the migration of particles in situations where inhomogeneous stress or shear fields are present, specifically in the pressure-driven flow of a suspension in a channel. This phenomenon is distinct from the migration of particles due to inertial forces, first observed by Segré \& Silberberg (1962). Here, the motion of the suspension is in the Stokes flow regime, i.e. $R e_{p} \ll 1$, where $R e_{p}$ is the

$\dagger$ Current address: India Institute of Science, Bangalore, India. 
Reynolds number based on the size of the suspended particles. This restriction to low Reynolds numbers is not an overly severe assumption, however, because it is satisfied in many practical situations of suspension processing. Furthermore, particle migration in inhomogeneous flows represents a new fundamental process and therefore deserves study in all regimes of Reynolds number.

Most previous theoretical and experimental studies of suspension flow have been on situations of uniform stress (or rate of strain) fields, e.g. narrow-gap Couette flow and shear flow between parallel plates. In such situations, the 'state' of the suspension is independent of position and there is thus no preferred position to be sought by the particles - an average particle moves about randomly as it follows along the mean suspension motion. This random motion is due to the chaotic nature of the particle evolution equations in concentrated suspensions. This random motion can give rise to a diffusive behaviour, and the shear-induced self-diffusivity of non-Brownian particles has been measured experimentally by Eckstein, Bailey \& Shapiro (1977) and Leighton $\&$ Acrivos (1987a), and has been studied via Stokesian dynamics simulations by Bossis \& Brady (1987), Phung \& Brady (1992) and Phung (1993).

The problem under consideration in the present work is different in that the concern here is on net migration of particles in a suspension exposed to inhomogeneous stress or shear fields. Inhomogeneous suspension flows are, of course, common in practical situations - flow in tubes or channels is probably the most common example. Moreover, most experimental measurements of suspension rheology are made in Couette viscometers, and this flow geometry may also give rise to an inhomogeneous flow field, unless the gap between the cylinders is small compared to their radii. Experiments on suspension flow in these geometries have been conducted by several investigators in recent years (Karnis, Goldsmith \& Mason 1966; Leighton \& Acrivos 1987 ; Hookham 1986; Koh 1991; Koh, Hookham \& Leal 1994; Sinton \& Chow 1991; Abbott et al. 1991).

The first evidence of shear-induced migration was provided by the experiment of Gadala-Maria \& Acrivos (1980), where they observed the steady decrease of the viscosity of a concentrated suspension in a Couette viscometer. Later, Leighton \& Acrivos $(1987 b)$ showed by further experiments that this was due to migration of particles from the Couette gap into the reservoir at the bottom, which was at a considerably lower shear rate. Migration of particles in this case was perpendicular to the plane of shear, but in the direction of the gradient in shear rate; in all other studies (on pressure-driven flow and wide-gap Couette flow), the gradient in shear rate was in the same direction as the shear rate. Among the studies on pressure-driven suspension flow, Hookham (1986) and Koh et al. (1994) have observed substantial particle migration accompanied by blunting (or flattening) of the velocity profile, while Karnis et al. (1966) and Sinton \& Chow (1991) report velocity blunting but no detectable migration of particles. The experiments of Abbott et al. (1991), in a wide-gap Couette device, show clearly the movement of particles away from the rotating inner rod, i.e. from regions of high to low shear rates.

Theoretical models for the migration process have been proposed by Leighton \& Acrivos (1987b) and Jenkins \& McTigue (1990). The former authors proposed a diffusion equation for particles, to be solved in conjunction with the continuity and momentum equations for the entire suspension. Jenkins \& McTigue on the other hand considered only the particle phase, and, in a manner akin to the treatment of dry granular materials, they propose conservation equations for mass, momentum and fluctuational energy for the particular phase. Thus, in their one-component system, there is no diffusion equation, but the particle concentration, 'temperature' (which is 
a measure of the fluctuational motion of the particles), and bulk velocity fields are set up so as to satisfy the continuity, momentum and energy equations. In this paper we develop a 'suspension balance' model which phenomenologically has similarities with model Jenkins \& McTigue propose, although the physics underlying the model is quite different.

From a fundamental standpoint, the mechanism for shear-induced migration has been controversial. As recently as in 1991, Abbott et al. attributed it to 'the existence of forces not described by Stokes equations', because it was implicitly assumed that the reversibility of the Stokes flow cannot produce irreversible motion. The mechanism put forward by Leighton \& Acrivos is that small-scale surface roughness of the particles leads to irreversible motion during inter-particle interactions; in a flow field with a gradient in shear rate, shear stress or particle concentration, net migration arises due to a greater number of interactions on one side of a particle than the other. However, surface roughness is not the sole mechanism and, as we demonstrate in this work, irreversible migration is produced even when the suspended particles are perfect hard spheres.

The serious disparities in the findings of the earlier investigations on the phenomenon of shear-induced migration and the ongoing speculations on the mechanism for migration provide motivation for further investigation on this subject, both experimental and theoretical. There is also a need, from the perspective of macroscopic modelling, to understand the mechanisms that cause microstructural changes in suspensions and to help develop the proper conservation and constitutive equations. The purpose of this study is two-fold. Firstly to verify earlier experimental findings on inhomogeneous suspension flow and rationalize the differences between them. To this end, we have performed dynamic simulations of the pressure-driven flow of a suspension in a rectangular channel by Stokesian Dynamics (Brady \& Bossis 1988), a method which accurately and efficiently computes the many-body long-range hydrodynamic interactions as well as the short-range lubrication interactions in Stokes flow. Since our simulations are for a suspension of perfect hard spheres at zero Reynolds number, factors such as inertial effects, surface roughness and nonhydrodynamic forces can be controlled. This provides a 'clean' experiment with which the results of earlier investigations can be compared. Secondly, it is hoped that this work will provide further mechanistic insight into the phenomenon of shear-induced particle migration and that the macroscopic transport equations we develop will provide a rational framework for modelling suspension flows.

In $\S 2$, we derive the timescale for achieving steady state in the pressure-driven flow of a viscous suspension, which is also valid in any other type of flow (e.g. wide-gap Couette flow), and show that it is considerably longer than that in the laminar flow of a pure Newtonian fluid. With this estimate of the timescale, we note that most measurements of earlier investigations were made within the transition length, i.e. before steady state was achieved, thus providing a probable explanation for the differences in their observations. Next, in $\S 3$, we outline the simulation method and its application to pressure-driven flow and discuss the importance of the suspension temperature in the dynamics of concentrated suspensions. The results of the simulations for various particle concentrations and channel widths are then presented in $\$ 4$, where we show substantial particle segregation and, concomitantly, blunting of the particle velocity field for all cases studied. The results of the simulations are compared with the experimental data of Karnis et al. (1966) and Koh et al. (1994) in \$5. We observe that most of the experiments of Koh et al. were not at steady state, but for those that were the concentration fields determined from our simulations are in qualitative (and 
perhaps quantitative) agreement. However, the velocity measurements reported by Koh $e t$ al. are in all cases considerably lower than the observations of this work. In $\S 6$, we compare the simulation results with the predictions of the 'diffusive flux' model of Leighton \& Acrivos and our 'suspension balance' model. We discuss the relative merits of the two models and show that the former can be derived as a special case of the latter. Finally, we conclude with a discussion of how the self-diffusion of a marked particle arises from the suspension balance equations, and present the criterion for a homogeneous shear flow to be stable to small perturbations.

\section{Timescale for shear-induced migration}

Karnis et al. (1966) conducted one of the earliest studies of inhomogeneous suspension flow under Stokes flow conditions. They measured velocity and concentration profiles of neutrally buoyant spheres in a Newtonian fluid when the suspension was pumped through a tube. For a range of the ratio $R / a$ ( $R$ and $a$ being the radii of the tube and the particles, respectively) and particle volume fraction, they reported substantial blunting of the velocity profiles. However, they did not detect any inhomogeneity in the particle concentration; the particles were evenly dispersed in the fluid, as they were at the entrance of the tube. More recently, Koh (1991) and Koh et al. (1994) conducted a systematic study of pressure-driven flow of a suspension through a rectangular channel. Using the laser Doppler velocimetry (LDV) technique, they measured velocities and concentrations of the suspended particles for a range of the channel width and particle concentration. Their results also show blunting of the velocity profile, but in contrast to the observations of Karnis et al., they always observed considerable inhomogeneity in the particle concentration due to migration of particles towards the centre of the channel.

A number of other experimental studies have confirmed the migration of particles from regions of high to low shear rate (Leighton \& Acrivos 1987 b; Abbott et al. 1991; Hookham 1986). However, the measurements differ in the extent of concentration inhomogeneity and the degree of velocity blunting. The differences between the observations may be attributed, in part, to inaccuracies in the experimental procedures and to the different measurement techniques that were used. For instance, Leighton \& Acrivos point out that the measurements of Karnis et al. may be flawed owing to the small number of tracer particles in their measurement volume. Sinton \& Chow cite flow-induced relaxation effects in their NMR imaging technique as a possible source of error in their concentration measurements. In what follows, however, we consider an aspect of the problem that has been overlooked in the majority of the earlier investigations - the time taken to achieve steady state, or alternatively, the 'transition length' in the channel (or tube) required for the flow to be fully developed. A simple analysis, similar to that used by Leighton \& Acrivos $(1987 \mathrm{~b})$, is given below that yields the time required to achieve steady state and the transition length.

Consider the flow of a suspension of particles of radii $a$ suspended in a Newtonian fluid driven by a pressure gradient (or by a uniform body force such as gravity) in a channel or tube of half-width $H$. The particles, which are initially dispersed homogeneously in the fluid, gradually migrate towards the centre of the channel (or tube) until they reach a steady configuration. Using the shear-induced-diffusion hypothesis of Leighton \& Acrivos $(1987 \mathrm{~b}$ ), the average distance travelled by the particles perpendicular to the direction of flow, $y$, in time $t$ is given by

$$
y=2(D t)^{1 / 2},
$$


$D$ being the shear-induced diffusivity. If the mean distance the particles must travel is taken to be of the order of the channel width, then the timescale for reaching steady state is

$$
t_{S S} \sim H^{2} /(4 D)
$$

Since the motion of the particles is driven purely by hydrodynamics,

$$
D=d(\phi) \dot{\gamma} a^{2},
$$

where $d(\phi)$ is a non-dimensional function of the particle volume fraction $\phi$ and $\dot{\gamma}$ is the shear rate. We estimate $\dot{\gamma}$ by its average value across the channel: $\dot{\gamma}=3\langle u\rangle / H$ for Poiseuille flow, where $\langle u\rangle$ is the average suspension velocity in the channel. Thus, (1) becomes

$$
t_{S S} \sim\left(\frac{H}{a}\right)^{3} \frac{a}{12 d(\phi)\langle u\rangle}
$$

The value of $d(\phi)$ can be estimated from measurements of the self-diffusivity in a suspension of hard spheres (Leighton \& Acrivos $1987 a$; Phung \& Brady 1992; Phung 1993; Phan \& Leighton 1994). (It might be more appropriate to use a collective as opposed to self-diffusivity here, but for scaling purposes this estimate is sufficient.) For dense suspensions $(\phi>0.3)$, the value of $12 d(\phi)$ is approximately 1 and hence $t_{S S} \sim$ $(H / a)^{3} a /\langle u\rangle$. Equation (3) can be expressed equivalently as the length along the channel required to reach steady state,

$$
\frac{L}{H} \sim \frac{1}{12 d(\phi)}\left(\frac{H}{a}\right)^{2}
$$

Note that this is just a characteristic length- (or time-) scale for the process and it may in general require several transition lengths before steady state is finally achieved. In contrast, the transition length in laminar flow of a homogeneous Newtonian fluid (within which the boundary layer reaches the centreline) is given by

$$
\frac{L}{H} \sim \frac{R e_{p}}{20}\left(\frac{H}{a}\right) .
$$

It should be clear that the time taken to achieve steady state can be much longer for a viscous suspension than for a homogeneous Newtonian fluid. Moreover, the $(H / a)^{2}$ scaling of the development length is rather stringent. In the experiments of Karnis et al. the actual length was not given, but the distance of $50 \mathrm{~cm}$ was reported for the translation of the microscope, so we use this as an estimate of the length. Using the smallest tube diameter reported of $0.2 \mathrm{~cm}, L / H \approx 250$, and from the values reported in table 1 of Karnis et al. there is one experiment at $(H / a)^{2} \approx 80$, one at 294 and the remainder range from 320 to 1700 . Thus, it is unlikely that any of the experiments of Karnis et al. were at steady state. In the experiments of Sinton \& Chow, the largest $L / H$ was 32.8 , while the smallest $(H / a)^{2}$ was 13520 ; clearly far from steady state. In Koh's experiments the particle size and channel length were kept fixed at $a=15 \mu \mathrm{m}$ and $L=12.7 \mathrm{~cm}$, respectively, and the channel width varied to change $H / a$. Of the four channel widths used $(0.15,0.0789,0.047$ and $0.026 \mathrm{~cm})$, only the last one is potentially at steady state. This long development length is clearly a possible reason for the disparity in the results of earlier investigations and is an important constraint to be taken into account in future studies. We show in $\$ 4$ that the above scaling for the steady-state time is borne out in the results of this investigation. The above scaling 
analysis is also applicable to other flow geometries and the conclusions are much the same.

\section{Simulation method}

Since the methodology of Stokesian Dynamics has been laid out in previous works (Brady \& Bossis 1988; Brady et al. 1988), only a brief account of the technique and its extension to pressure-driven flow is given here. The method exploits the fact that hydrodynamic interactions among particles can be decomposed into long-range mobility interactions and short-range lubrication interactions. The long-range interactions are first computed as a moment expansion about the particle centres of the force densities acting on their surfaces. The zeroth moment is simply the net rorce acting on a particle; the first moment can be decomposed into the torque and the stresslet; higher moments in the series are discarded. This truncated multipole expansion, in conjunction with Faxén's laws, is used to form the grand mobility tensor, $\mathscr{M}^{\infty}$. A unit cell with a finite number of particles is replicated periodically throughout all space and the long-range interactions between all particles are then summed using the Ewald technique to hasten the convergence. Once constructed, the grand mobility tensor is inverted to yield the far-field approximation to the grand resistance tensor $\mathscr{R}^{\infty}$. Though $\mathscr{M}^{\infty}$ is pairwise additive, on inversion all infinite reflections are computed. Thus $\mathscr{R}^{\infty}$ captures the many-body interactions among particles. Finally, the near-field lubrication interactions are added to the resistance tensor in a pairwise-additive manner to form the grand resistance tensor $\mathscr{R}$.

The grand resistance tensor relates the vector of hydrodynamic forces and torques $(\boldsymbol{F})$ and stresslets $(\boldsymbol{S})$ on the particles in a unit cell to their respective velocities and the rate of strain:

$$
\left(\begin{array}{l}
\boldsymbol{F} \\
\boldsymbol{s}
\end{array}\right)=-\mathscr{R} \cdot\left(\begin{array}{c}
\boldsymbol{u}-\langle\boldsymbol{u}\rangle \\
-\langle\boldsymbol{e}\rangle
\end{array}\right)
$$

where

$$
\mathscr{R}=\left[\begin{array}{ll}
\boldsymbol{R}_{F U} & \boldsymbol{R}_{F E} \\
\boldsymbol{R}_{S U} & \boldsymbol{R}_{S E}
\end{array}\right] .
$$

In $(6)\langle\boldsymbol{u}\rangle$ is the average velocity of the suspension (particles and fluid) and $\langle\boldsymbol{e}\rangle$ is its rate of strain. The $6 N$ vector $u$ denotes the translational and rotational velocities of all particles. The subscripts on the resistance tensors denote the respective couplings; e.g. the subscript in $\boldsymbol{R}_{F U}$ denotes the coupling between force and velocity. The resistance tensors are functions of the particle configuration only. They are computed at each time step and substituted in (6) to solve for the particle velocities if the forces are known, or vice versa. External or inter-particle forces acting on the particles are also functions only of the particle configuration and can be computed for each configuration. The evolution equation for the particles then follows from the balance of hydrodynamic and other forces at low Reynolds number; particle inertia is not important.

To simulate pressure-driven flow, the Stokesian Dynamics method as used previously needs to be adapted to allow for the presence of walls. One approach for this, which was taken by Durlofsky \& Brady (1989), is to divide the walls into patches in the integral formulation of Stokes equations. The far-field interactions are then computed by assuming that the force density over each patch is a constant. The near-field sphere-wall interactions can be included just as is done for sphere-sphere interactions. An alternative method is to designate some of the spheres in the unit cell as wall particles; they are placed in a plane and required to all move at the same velocity (e.g. 


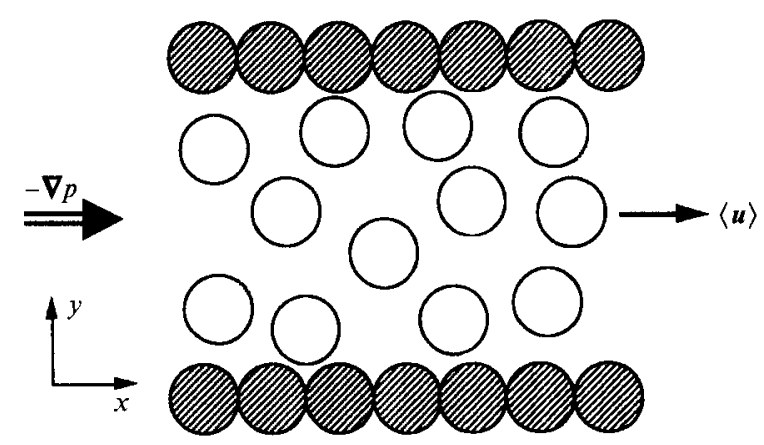

FIGURE 1. The unit cell for simulations of pressure-driven flow in a channel. The hatched circles represent wall particles and the open circles the interior particles. The wall particles are kept stationary while the interior particles are free to move in the $x$-and $y$-directions.

zero), thus forming a 'bumpy' wall, as illustrated in figure 1. Though there will be a small quantitative effect of having bumpy walls, the qualitative behaviour of the system is expected to remain unchanged. The interior particles, on the other hand, are free to move in any direction. In this method, the velocities of the wall particles and the forces on the interior particles are prescribed, leaving the velocities of the interior particles and the forces on the wall particles to be determined. The bulk shear rate $\langle\boldsymbol{e}\rangle$ is set to zero as the flow is driven by the pressure gradient in the suspension. If we specify a fixed average suspension velocity, $\langle\boldsymbol{u}\rangle$, then a pressure gradient will be set up so as to satisfy the global conservation of momentum. In the present case, we specify that the wall particles are stationary $\left(\boldsymbol{u}^{W}=0\right)$, so the force required to hold these particles stationary will balance the pressure gradient needed to maintain the non-zero average $\langle\boldsymbol{u}\rangle$. Note also that as a consequence of the unit cell being replicated in all directions, we have a series of parallel channels through which the fluid and freely suspended particles are pumped. This technique is quite easy to implement as the computational scheme used in earlier studies of unbounded systems (Phung \& Brady 1992) could be used here with few modifications.

Since the bulk rate of strain is zero, the hydrodynamic force on the particles for this system can be reduced from (6) to

$$
\begin{aligned}
& F^{W}=\boldsymbol{R}_{F U}^{W W} \cdot\langle\boldsymbol{u}\rangle-\boldsymbol{R}_{F U}^{W I} \cdot\left(\boldsymbol{u}^{I}-\langle\boldsymbol{u}\rangle\right), \\
& \boldsymbol{F}^{I}=-\boldsymbol{R}_{F U}^{I I} \cdot\left(\boldsymbol{u}^{I}-\langle\boldsymbol{u}\rangle\right)+\boldsymbol{R}_{F U}^{I W} \cdot\langle\boldsymbol{u}\rangle .
\end{aligned}
$$

Here the superscripts $W$ and $I$ refer to the wall and interior particles, respectively, and $\boldsymbol{R}_{F U}^{W W}$ represents the resistance tensor for interactions between wall particles, $\boldsymbol{R}_{F U}^{W I}$ for wall-interior interactions and so on. Hence, the governing equation for the motion of the interior particles is simply

$$
F^{I}+F^{o}=0,
$$

where $F^{o}$ is the non-hydrodynamic interparticle force exerted on the interior particles. With (9) we then have for the evolution of particle positions

$$
\frac{\mathrm{d} \boldsymbol{x}^{I}}{\mathrm{~d} t}=\langle\boldsymbol{u}\rangle+\left(\boldsymbol{R}_{F U}^{I I}\right)^{-1} \cdot\left(\boldsymbol{F}^{O}+\boldsymbol{R}_{F U}^{I W} \cdot\langle\boldsymbol{u}\rangle\right) .
$$

The wall particles remain stationary $\left(\boldsymbol{u}^{W}=0\right)$ relative to the suspension average velocity and the external forces required to hold them fixed are equal and opposite to the hydrodynamic forces from (8),

$$
\boldsymbol{F}_{e x t}^{W}=-\boldsymbol{F}^{W}=-\boldsymbol{R}_{F U}^{W W} \cdot\langle\boldsymbol{u}\rangle+\boldsymbol{R}_{F U}^{W I} \cdot\left(\boldsymbol{R}_{F U}^{I I}\right)^{-1} \cdot\left(\boldsymbol{F}^{O}+\boldsymbol{R}_{F U}^{I W} \cdot\langle\boldsymbol{u}\rangle\right) .
$$


The pressure gradient is then found from an overall momentum balance for a unit cell,

$$
\nabla\langle p\rangle=n_{W}\left\langle\boldsymbol{F}_{e x t}^{W}\right\rangle
$$

where $n_{W}$ is the number density of fixed wall particles.

In the governing equations, time has been scaled with $a /\langle u\rangle$, particle velocities with $\langle u\rangle$ and all forces with $6 \pi \eta a\langle u\rangle$. Here, $\langle u\rangle$ is the average suspension velocity (over the whole channel) in the flow direction, $a$ is the radius of the spheres and $\eta$ is the viscosity of the suspending fluid. It should be noted here that the average suspension velocity appearing in (8) (13) includes the wall particles as part of the suspension. The average suspension velocity needed for comparison with experiment is that for the flow of material between the channel walls and must be calculated by disregarding the volume occupied by the wall particles. This corrected average velocity, equal to $\langle u\rangle(1+a / H)$, is the one used to scale the variables described above. The simulations were carried out until steady states were achieved, i.e. until the velocity and density fields no longer changed with time.

Equation (11), which is a set of coupled, nonlinear ordinary differential equations for the particle positions, gives rise to deterministic chaos. It is this chaotic motion of the particles that is responsible for the shear-induced diffusion in homogeneous shear flow and particle migration in inhomogeneous flows. However, chaotic (or irreversible) motion sets in only beyond the 'domain of determinism' for the system of equations and, within this time domain, the reversibility of Stokes flow applies and particle paths are indeed reversible. Chaotic motion is not produced when there are just two particles in the system, however, as a two-body interaction is reversible; three or more body interactions are needed to produce chaotic motion. In this light, the assumptions of Abbott et al. that irreversible migration is produced by non-Stokes forces and of Leighton \& Acrivos that it is produced by surface roughness, are not necessary.

As a measure of the fluctuational motion of particles in a suspension arising from this chaos, we introduce the 'suspension temperature' (henceforth referred to as temperature for brevity), defined as

$$
T=\left\langle\boldsymbol{u}^{\prime} \cdot \boldsymbol{u}^{\prime}\right\rangle_{p}
$$

with $\boldsymbol{u}^{\prime}$ being the velocity fluctuation of a particle about its local mean velocity and the angle brackets denoting an ensemble average over all particles. $\dagger$ The temperature is a quantity of interest because the fluctuational motion of particles is closely related to the shear-induced diffusivity.

By definition, the long-time tracer diffusivity of a particle is related to the meansquare displacement and the velocity fluctuation autocorrelation function as

$$
D \equiv \lim _{t \rightarrow \infty} \frac{1}{2} \frac{\mathrm{d}}{\mathrm{d} t}\left\langle\left(\boldsymbol{x}-\langle\boldsymbol{x}\rangle_{p}\right)^{2}\right\rangle_{p}=\int_{0}^{\infty}\left\langle\boldsymbol{u}^{\prime}(0) \cdot \boldsymbol{u}^{\prime}(t)\right\rangle_{p} \mathrm{~d} t .
$$

Since $\left\langle\boldsymbol{u}^{\prime}(0) \cdot \boldsymbol{u}^{\prime}(0)\right\rangle_{p}$ is equal to the temperature, the diffusivity scales as the temperature times the correlation time for the velocity fluctuations, $\tau$. If, as in kinetic theory, we take the correlation time as the characteristic size of the particles divided by the r.m.s. of the velocity fluctuations, then $\tau \sim a / T^{1 / 2}$ and hence

$$
D \sim a T^{1 / 2} \text {. }
$$

$\dagger$ A more general measure of the velocity fluctuations is a tensorial temperature, defined as $\left\langle\boldsymbol{u}^{\prime} \boldsymbol{u}^{\prime}\right\rangle_{p}$, which is appropriate in situations where the magnitude of the velocity fluctuations may have a directional dependence. Since the purpose of introducing the temperature in this work is to qualitatively explore its importance in determining the suspension microstructure, we use the scalar form given by (14) and defer a more rigorous approach to later studies. 
In homogeneous shear flow, $T \sim \dot{\gamma}^{2} a^{2}$ since the shear rate is the only timescale in the problem. Hence,

$$
D \sim T \tau \sim \dot{\gamma} a^{2}
$$

and we recover the same scaling as in the shear-induced diffusion mechanism of Leighton \& Acrivos (see (2)). (Note we have not specified any $\phi$-dependence in the connection between $D$ and $T$, as this requires detailed analysis of particle interactions, especially at low $\phi$.) Though the scaling with $\dot{\gamma}$ is the same, the diffusivity should be viewed as being proportional to the square root of the temperature rather than to the shear rate. This difference is important from a fundamental point of view and its significance will become clear when we discuss the results of the simulations in $\S 4$ and the theories in $\S 6$. In homogeneous shear flow, the shear rate is independent of position and so is the temperature; however, the shear rate and temperature in pressure-driven flow vary with position. Since the characteristic shear rate in pressure-driven flow is $\langle u\rangle / H$, we have scaled the temperature with $\langle u\rangle^{2}(a / H)^{2}$ in this work.

Owing to the constant fluctuational motion of the particles, the pointwise velocity, concentration and temperature fields are not smooth functions of position or time. To smooth the fluctuations, the particle velocities and concentrations were averaged over space and time as follows: the channel was first divided into several sections of roughly equal width. The areal fraction and area-weighted particle average velocity in each of these sections were then calculated. These quantities were then averaged over a period of time long enough such that their time fluctuations were smoothed out. Thus, the average particle velocity at a point is given by,

$$
\boldsymbol{u}_{\text {avg }}(y)=\overline{\langle\langle\boldsymbol{u}\rangle\rangle}
$$

where the double angle brackets denote area-weighted averaging within each section and the overline denotes time averaging. For all simulations listed in table 1 , the width of the averaging sections was chosen to be half a particle radius and the time averaging was carried out over a dimensionless time of 1200 .

Now, replacing the ensemble average by volume and time averages in the definition of the temperature requires more care because this can be done in two ways:

$$
T=\overline{\boldsymbol{u}^{2}-\langle\langle\boldsymbol{u}\rangle\rangle^{2}} \text { or } T=\overline{\boldsymbol{u}^{2}}-(\overline{\langle\langle\boldsymbol{u}\rangle\rangle})^{2} \text {. }
$$

The two definitions are, of course, identical when the average particle velocity at any point does not vary with time. However, the only velocity that is specified to be a constant in the simulations is the average velocity of the entire suspension in the channel. The average particle velocity at any point can and does vary with time as, for instance, when the entire group of particles fluctuates together in the flow direction about its mean position. This concerted movement of particles causes fluctuations in the pressure gradient (a 'pump noise') which, while contributing to the velocity fluctuations, does not lead to any relative motion of the particles. Therefore, to filter out this noise and calculate a temperature based on the fluctuational velocity of individual particles about the instantaneous average velocity, the former definition of temperature in (18) was chosen. Another issue regarding the calculation of the temperature pertains to the bumpy walls; in many cases, a few particles are 'captured' by the strong lubrication forces with the wall particles and translate along the walls. In doing so, they execute a bumpy motion whose contribution to the temperature is large compared to the temperature in the interior of the channel (indeed it scales as $\langle u\rangle^{2}$ rather than $\left.\langle u\rangle^{2}(a / H)^{2}\right)$. Since this contribution to the temperature is purely an artifact of having the walls composed of particles (rather than having a flat wall), it is ignored by neglecting the velocity fluctuations of particles that are closer than a distance of 0.01 to any wall particle. 
For the full three-dimensional problem, there are three unknown velocities (or forces), three angular velocities (torques) and five strain rates (stresslets) for every particle. The grand mobility tensor is therefore of size $11 N \times 11 N$, the inversion of which requires $O\left((11 N)^{3}\right)$ operations. For a system with 50 or more particles, this demanded more computational time than could be afforded, particularly because of the long times required to achieve steady state (see $\S 4$ ). To reduce the computational cost, the movement of the interior particles was restricted to the $x$ - and $y$-directions. To this end, a unit cell was chosen such that its $z$-dimension was equal to one particle diameter (see figure 1). This monolayer cell was then periodically replicated in all directions - thus, the particles were in close contact with their periodic neighbours in the $z$-direction, but the chains of particles were free to move in the $x$-and $y$-directions. Sample runs were also made with the layer of particles spaced further apart in the $z$ direction than one diameter; there were no significant differences. There are now only $6 \mathrm{~N}$ unknowns in the problem and the number of particles in the unit cell is also significantly fewer, representing computational savings of a factor of at least $1000 \mathrm{over}$ the full three-dimensional problem. For the monolayer, the volume fraction of particles is simply $\frac{2}{3}$ times the areal fraction $\phi_{A}$. All results in $\S 4$ are given in terms of the areal fraction of particles.

When integrating (11), the time step $\Delta t$ has to be small enough that overlap of particles does not occur. On the other hand, choosing too small a time step increases the computational time of the simulation. Therefore, the largest time step that did not result in particle overlap was determined by a procedure of trial-and-error; this was 0.01 for all the simulations, with the exception of simulation B1, for which it was set to 0.005 . The far field interactions that contribute to the grand mobility tensor $\mathscr{M}^{\infty}$ change appreciably only when the inter-particle distances change by more than a particle radius. Hence, the grand mobility matrix was updated and inverted only at time intervals of 1 . The computations were performed on a CRAY-YMP and an IBM RISC-6000 workstation. A simulation with 51 particles required about 3 hours of CPU time on the former and about 9 hours on the latter to reach a dimensionless time of 1000 .

\section{Results}

The only parameters in the flow of a suspension of monodisperse particles in a channel are the areal fraction of particles, $\phi_{A}^{b}$, and the dimensionless channel width, $H / a$. (The value of $H$ for the 'bumpy' channel is taken to be the shortest distance between the walls.) One of the major objectives of this work is to explore the influence of these two parameters. Table 1 lists the parameter values and other quantities of interest for the simulations discussed in this section. The input parameters in the problem, namely the bulk particle concentration, the number of particles in the unit cell, the number of wall particles (which also determines the length of the cell - see figure 1) and the channel width are given in columns 2, 3, 4 and 5 respectively. A shortrange repulsive force between interior particles was used in many of the simulations (cf. $\S 4.1$ ) and the range of this force is given in column 6. In columns 7 and 8 are the velocity and areal fraction at the channel centre, quantities which reflect the degree of velocity blunting and particle migration. Column 9 lists the average particle velocity in the channel, column 10 the dimensionless particle flux in the channel, column 11 lists the pressure gradient required to pump the suspension (normalized by the pressure gradient for pure fluid) and column 12 gives the total time for each simulation.

All simulations are for monodisperse non-Brownian hard spheres in the absence of 


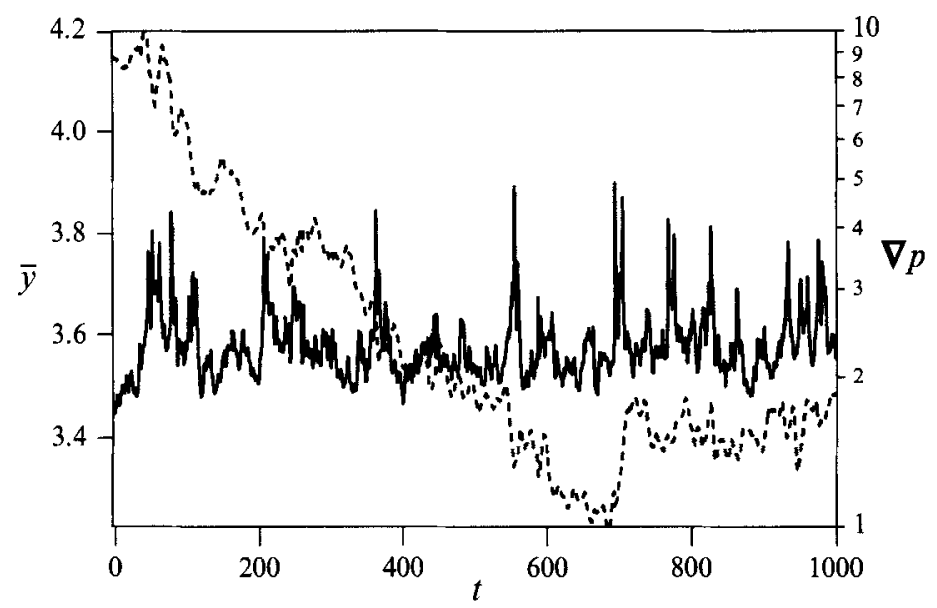

FIGURE 2. Time traces of the average distance of particles from the centreline (----) and the pressure gradient required to pump the suspension (-) for a bulk areal fraction $\phi_{A}^{b}$ of 0.4 and a channel width $H / a$ of 18.32 .

$\begin{array}{cccccccccccr}\text { Simulation } & \phi_{A}^{b} & N & N_{w} & \frac{H}{a} & \tau & \frac{u^{\max }}{\langle u\rangle} & \phi_{A}^{\max } & \langle u\rangle_{p} & Q_{p} & \frac{\boldsymbol{\nabla}\langle p\rangle}{\boldsymbol{\nabla} p_{0}} & t_{\text {sim }} \\ \text { A } & & & & & & & & & & & \\ \text { B1 } & 0.4 & 51 & 9 & 14.02 & 1000 & 1.37 & 0.71 & 0.954 & 1.19 & 2.44 & 2400 \\ \text { B2 } & 0.4 & 51 & 9 & 18.32 & 100 & 1.32 & 0.64 & 0.980 & 1.15 & 2.37 & 4800 \\ \text { B3 } & 0.4 & 51 & 9 & 18.32 & 1000 & 1.34 & 0.71 & 0.975 & 1.18 & 2.13 & 4800 \\ \text { B4 } & 0.4 & 51 & 9 & 18.32 & 10000 & 1.35 & 0.70 & 0.973 & 1.18 & 2.08 & 4800 \\ \text { B5 } & 0.4 & 102 & 18 & 18.32 & 1000 & 1.35 & 0.68 & 0.976 & 1.18 & 2.05 & 4800 \\ \text { C } & 0.4 & 61 & 7 & 30.29 & 1000 & 1.33 & 0.78 & 0.988 & 1.18 & 2.15 & 4800 \\ \text { D } & 0.4 & 79 & 7 & 40.40 & 1000 & 1.30 & 0.83 & 0.993 & 1.17 & 2.02 & 36000 \\ \text { E } & 0.3 & 26 & 7 & 14.02 & 1000 & 1.42 & 0.67 & 0.943 & 1.23 & 1.89 & 2400 \\ \text { F } & 0.3 & 41 & 9 & 18.32 & 1000 & 1.37 & 0.63 & 0.967 & 1.24 & 1.61 & 9600 \\ \text { G } & 0.3 & 48 & 7 & 30.29 & 1000 & 1.36 & 0.72 & 0.974 & 1.23 & 1.62 & 29000 \\ \text { H } & 0.3 & 61 & 7 & 40.40 & 1000 & 1.35 & 0.63 & 0.982 & 1.18 & 1.75 & 32000 \\ \text { I } & 0.15 & 25 & 9 & 18.32 & 1000 & 1.46 & 0.45 & 0.801 & 1.29 & 1.32 & 7200 \\ \text { J } & 0.45 & 56 & 9 & 18.32 & 1000 & 1.32 & 0.74 & 0.976 & 1.17 & 2.37 & 4800\end{array}$

TABLE 1. List of all simulations discussed is this work. Columns 2-6 list the input parameters for the problem and the rest are output parameters that show the degree and effect of particle migration.

external forces. However, with the exception of simulation, B1, a repulsive interaction between the interior particles was used in all cases (cf. §4.1). The initial particle configurations were generated by a Monte-Carlo technique; the interior particles were first placed in a regular array between the walls and then successively given small random displacements until a uniform distribution was achieved.

As a illustrative case, we first discuss the results from simulation B1 so that the features common to all the simulations may be noted. For this case, the bulk areal fraction of particles is 0.4 and the width of the channel is 18.32 particle radii. Figure 2 shows the time traces of the dimensionless pressure gradient $\nabla\langle p\rangle$ and the average distance of the particles from the channel centre $\bar{y}$. While the former is a measure of the bulk response of the suspension to pressure-driven flow, the latter traces the evolving inhomogeneity of the microstructure $-\bar{y}$ decreases as the particles move 

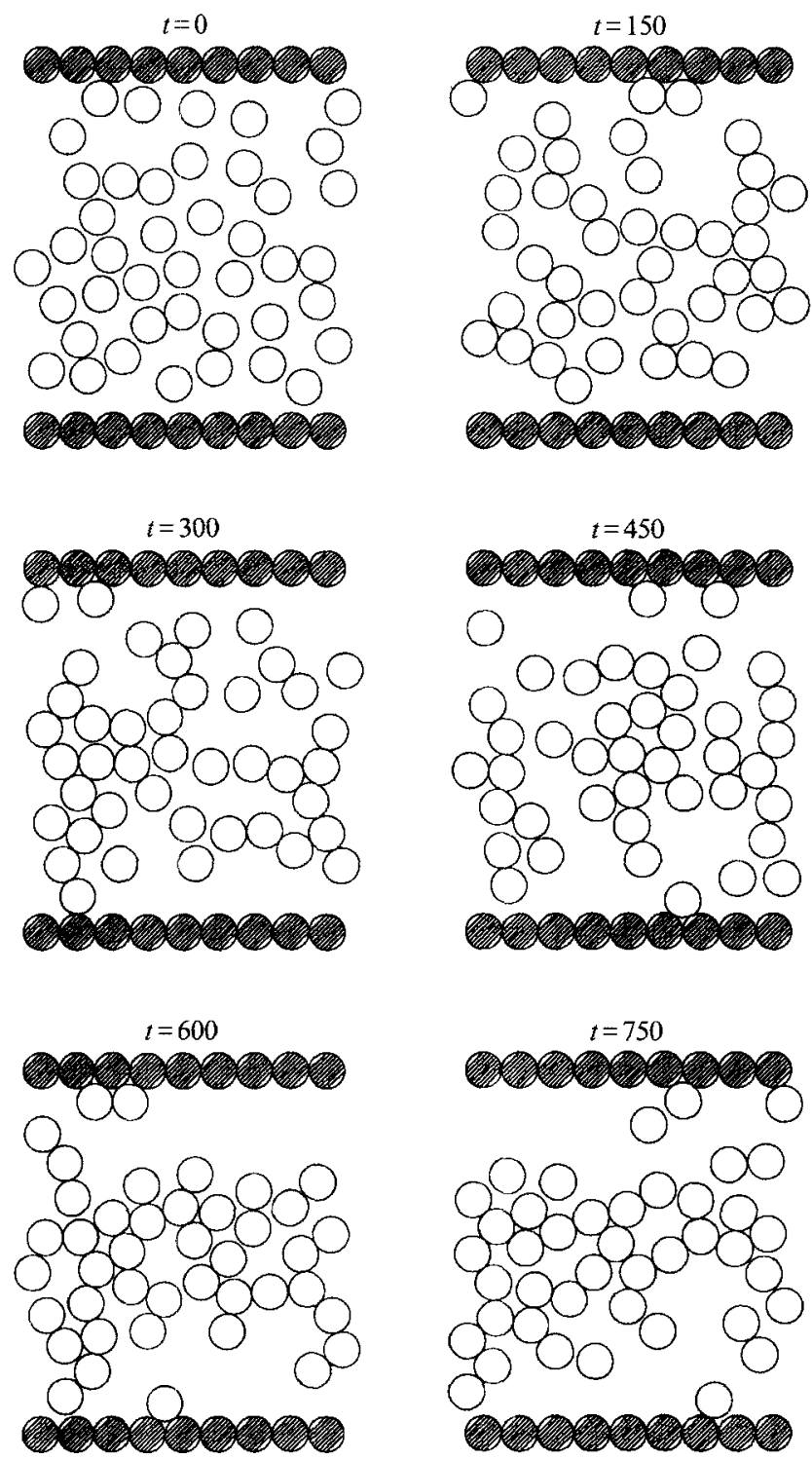

FigURE 3. Snapshots of particle configuration at time intervals of 150 for $\phi_{A}^{b}=0.4$ and $H / a=18.32$. The hatched circles represent wall particles and the open circles the interior particles. Note the gradual migration of particles towards the centre of the channel.

towards the centre. It is clear that in these simulations the microstructure takes a much longer time to reach steady state than the pressure gradient. One should note however, that in the simulations the total material flux and the number of particles are held constant, while in many laboratory experiments the particle flux is held fixed, and thus the pressure drop may vary as the microstructure evolves.

The occasional peaks in the pressure gradient correspond to instances when particle clusters are large enough to span the width of the channel. To maintain a constant flow rate, a larger pressure gradient is required at these moments. It is also interesting to note that the pressure gradient increases initially, as the particles migrate away from the walls. This is contrary to one's intuitive expectation, as the movement of particles 
from the walls will reduce the local viscosity of the suspension adjacent to the walls. However, migration of particles away from the walls also leads to a rise in the shear rate there and this appears to outweigh the effect of a smaller local suspension viscosity.

Figure 3 presents snapshots of particle configuration in the unit cell for Simulation B1 from the start till $t=750$ at time intervals of 150 . The hatched circles here represent wall particles. The evolution of the microstructure is clearly visible, with the particles dispersed evenly at the start of migrating steadily towards the centre.

The density profile in figure $4(a)$ shows pronounced particle segregation in the channel, the areal fraction at the centre being slightly over 0.6 . The small concentration peaks near the walls are due to the excluded volume effect of the walls; particle centres cannot be closer than one radius from the wall and this exclusion from regions less than one radius results in a slight enhancement of density at one radius. The clustering of particles around the centre of the channel causes a blunting of the particle velocity profile as shown in figure $4(b)$. A higher particle concentration around the centre leads to a larger suspension viscosity; since the shear stress varies linearly with position, the shear rate is diminished near the channel centre. The thick line in figure $4(b)$ is the parabolic velocity profile of a Newtonian fluid, shown for comparison. The suspension temperature profile in figure $4(c)$ (solid line) shows that, except for the layers adjacent to the walls where particles are constrained from motion, the temperature varies with the local shear rate - it is maximum at the periphery and minimum at the centre of the channel. However, it must be noted that the fluctuation velocity does not vanish at the centre and the particles there retain their ability to sample the space around their mean position. In other words, the particle diffusivity at the centreline is non-zero, as elsewhere in the channel. This is an important observation in view of the fact that the theories of Leighton \& Acrivos and Phillips et al. (1992) have assumed that the shearinduced diffusivity is linearly proportional to the local shear rate. The results of this work demonstrate a more complex relationship between the diffusivity and the local shear rate, simply because the temperature and therefore diffusivity are finite at the centre of the channel, while the shear rate vanishes; further discussion of this issue is given in $\S 6$. The dashed curves in figure $4(c)$ are the mean-square velocity fluctuations in the $x$-and $y$-directions; they show that there is an anisotropy in the temperature, although the magnitudes and spatial variations in both directions are comparable.

\subsection{Effect of inter-particle forces}

As mentioned earlier, most of the simulations listed in table 1 were carried out with short-range repulsive forces included in the interactions between the interior particles for at least part of the simulation period; there was no force between interior and wall particles. The forces were chosen to be of the form used by Durlofsky \& Brady (1989),

$$
\boldsymbol{F}_{\alpha \beta}=F_{0} \frac{\tau \mathrm{e}^{-\tau \epsilon}}{1-\mathrm{e}^{-\tau \epsilon}} \boldsymbol{e}_{\alpha \beta}
$$

where $F_{\alpha \beta}$ is the force exerted on sphere $\alpha$ by sphere $\beta, F_{0}$ is a constant representing the magnitude of the force, $\tau$ is related to the range, $\epsilon$ is the spacing between the surfaces of spheres $\alpha$ and $\beta$ and $e_{\alpha \beta}$ is the unit vector connecting the sphere centres. For all cases where this repulsive interaction was employed, the value of $\tau$ was set at 1000 , with the exceptions of simulations B2 and B4, where it was 100 and 10000 respectively; the product $F_{0} \tau$ was always equal to unity. With $\tau=1000$ and $F_{0}=0.001$, (19) gives a repulsive force of magnitude unity for $\epsilon \approx 0.00069$.

To determine the influence of these short-range interactions on particle migration, simulations B2-B4 were performed for different values of $F_{0}$ and $\tau$, but under otherwise 

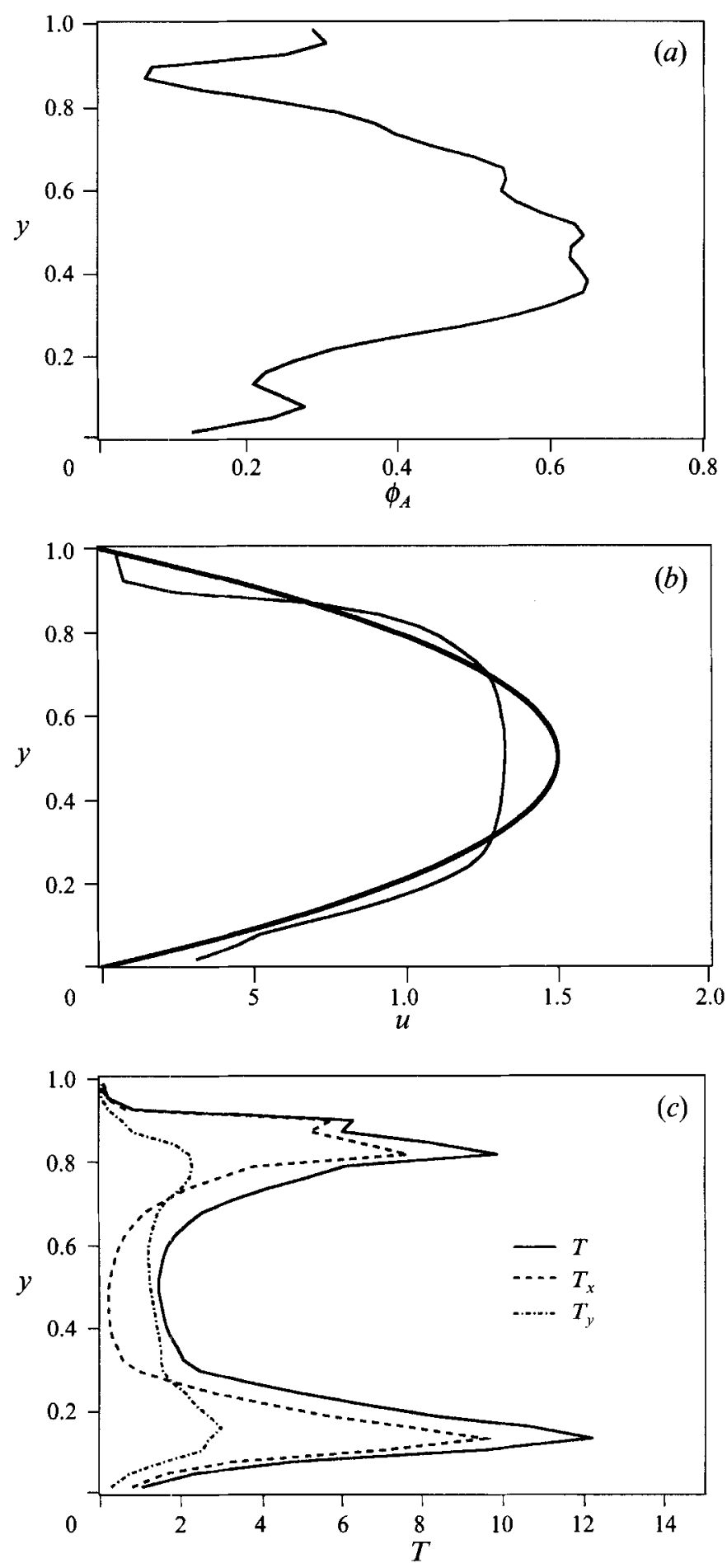

FIGURE 4. The steady-state concentration $(a)$, velocity $(b)$ and suspension temperature $(c)$ profiles for $\phi_{A}^{b}=0.4$ and $H / a=18.32$. The dark line in $(b)$ is the parabolic velocity profile for a Newtonian fluid. 


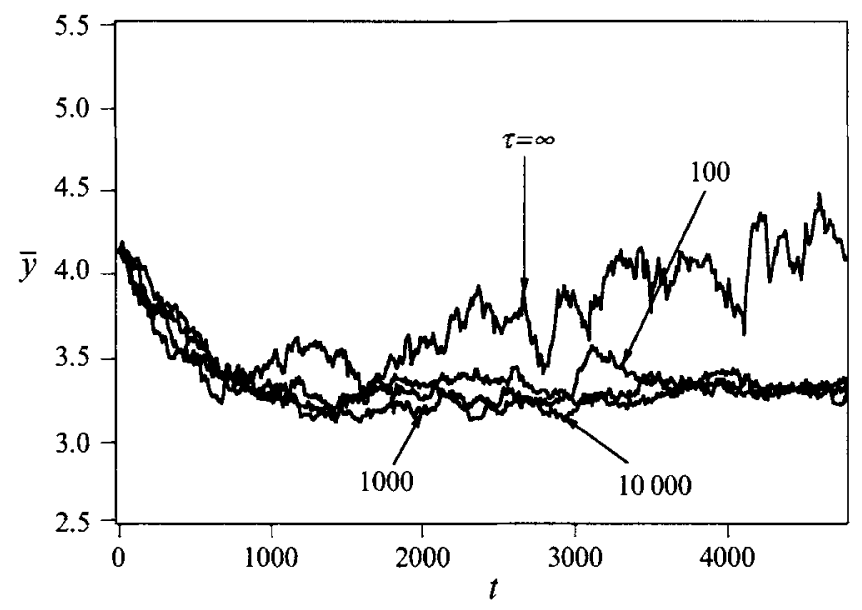

FIGURE 5. The time trace of the average distance of particles from the centreline for simulations B1-B4, each for a different range (and strength) of the repulsive inter-particle force. Note the similarity in the behaviour for the cases with non-zero inter-particle force.

identical starting conditions. The time trace of $\bar{y}$ for these simulations in figure 5 shows that the behaviour for the cases with non-zero repulsive forces is strikingly similar; the steady-state value of $\bar{y}$ and the relaxation time for the migration of particles are almost identical for the three cases. The evolution of $\bar{y}$ with time for simulation B1, for which no inter-particle force was assumed, is somewhat different. Though the initial behaviour is similar, showing a relaxation response almost identical to the other three cases, the value of $\bar{y}$ rises and falls back repeatedly. These oscillations in $\bar{y}$ are caused by the formation of clusters that span the width of the channel. When they subsequently break up, the particles at the centre of the channel are displaced en masse away from the axis, leading to a rise in $\bar{y}$, and a fall when the cluster of particles moves back towards the centre. The skewed particle distribution resulting from this phenomenon is apparent from the density profile in figure $6(a)$.

Thus, the inter-particle repulsive force prevents the formation of large clusters by counteracting the strong lubrication forces which tend to keep particles together once they are in close proximity. This enables a more compact arrangement of the particles at the centre, resulting in the density profile being more peaked in the presence of an inter-particle repulsive force, as shown in figure 6(a). The velocity and temperature fields (figure $6 b, c$ ) remain unchanged regardless of whether or not the inter-particle force is present - i.e. they are uninfluenced by small changes in the microstructure. The same is also true of the mean-square velocity fluctuations in the $x$-and $y$-directions. The similarity of the fields over a three-decade variation in the range of repulsive force implies that this interaction has no effect other than that mentioned above.

In reality, the strong lubrication forces may be counteracted by mechanisms such as Brownian motion, surface roughness of particles, etc., thus providing ways in which the clusters may be broken. The purpose of the repulsive force is to qualitatively model these mechanisms; it has the effect of breaking large clusters, while leaving the longrange interactions unaltered. It is important to note that the net migration of particles is the same regardless of whether or not there are repulsive forces between particles, and therefore these forces, or the irreversible interactions (surface roughness etc.) postulated by other workers, are not essential for the phenomenon of shear-induced migration. 


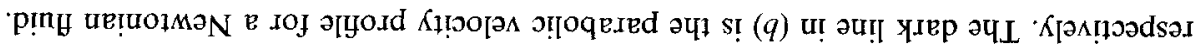

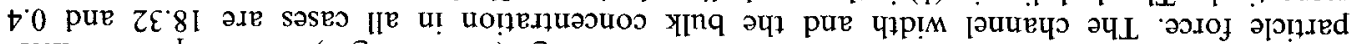

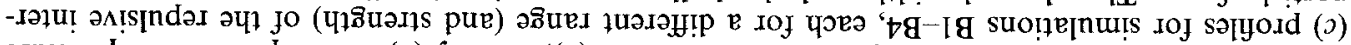

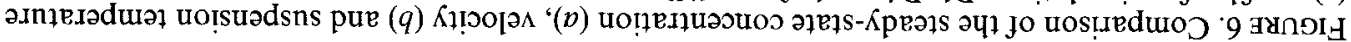
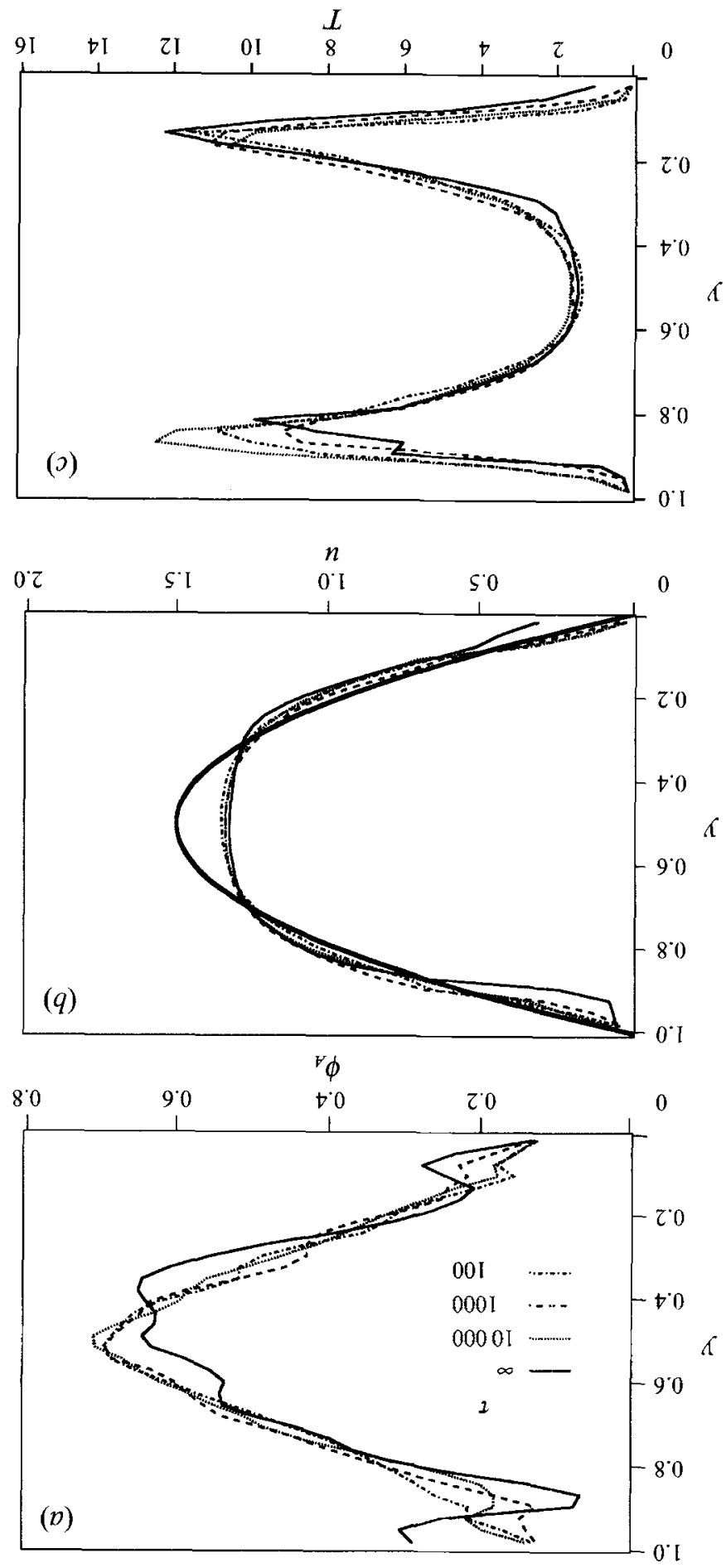

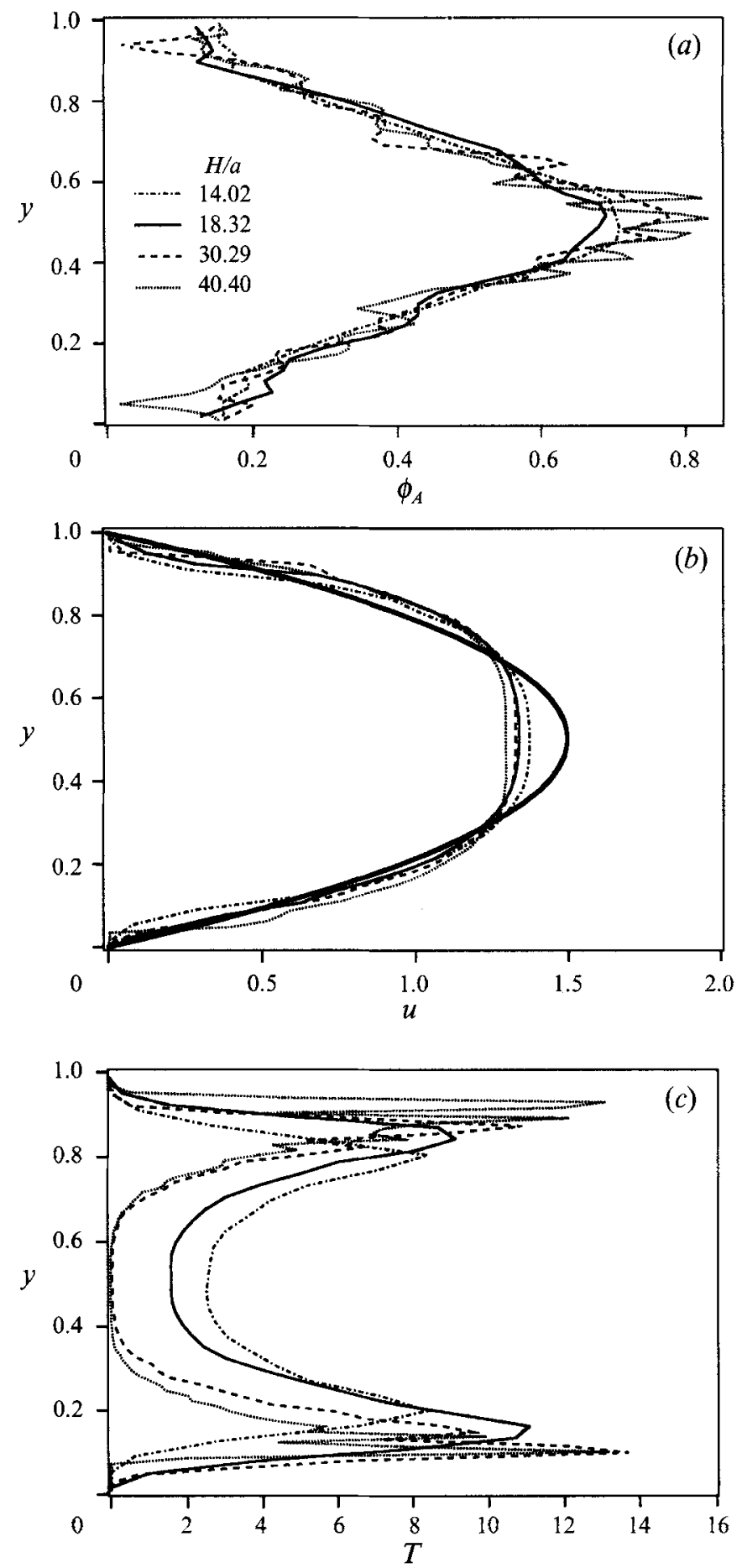

FIGURE 7. The steady-state concentration $(a)$, velocity $(b)$ and suspension temperature $(c)$ profiles for the simulations with $H / a=14.02,18.32,30.29$ and 40.40 and $\phi_{A}^{b}=0.4$ (simulations $\mathrm{A}, \mathrm{B} 3, \mathrm{C}$ and D). The dark line in $(b)$ is the parabolic velocity profile for a Newtonian fluid. The inter-particle force in all cases was such that $\tau=1000$ and $F_{0}=0.001$. 

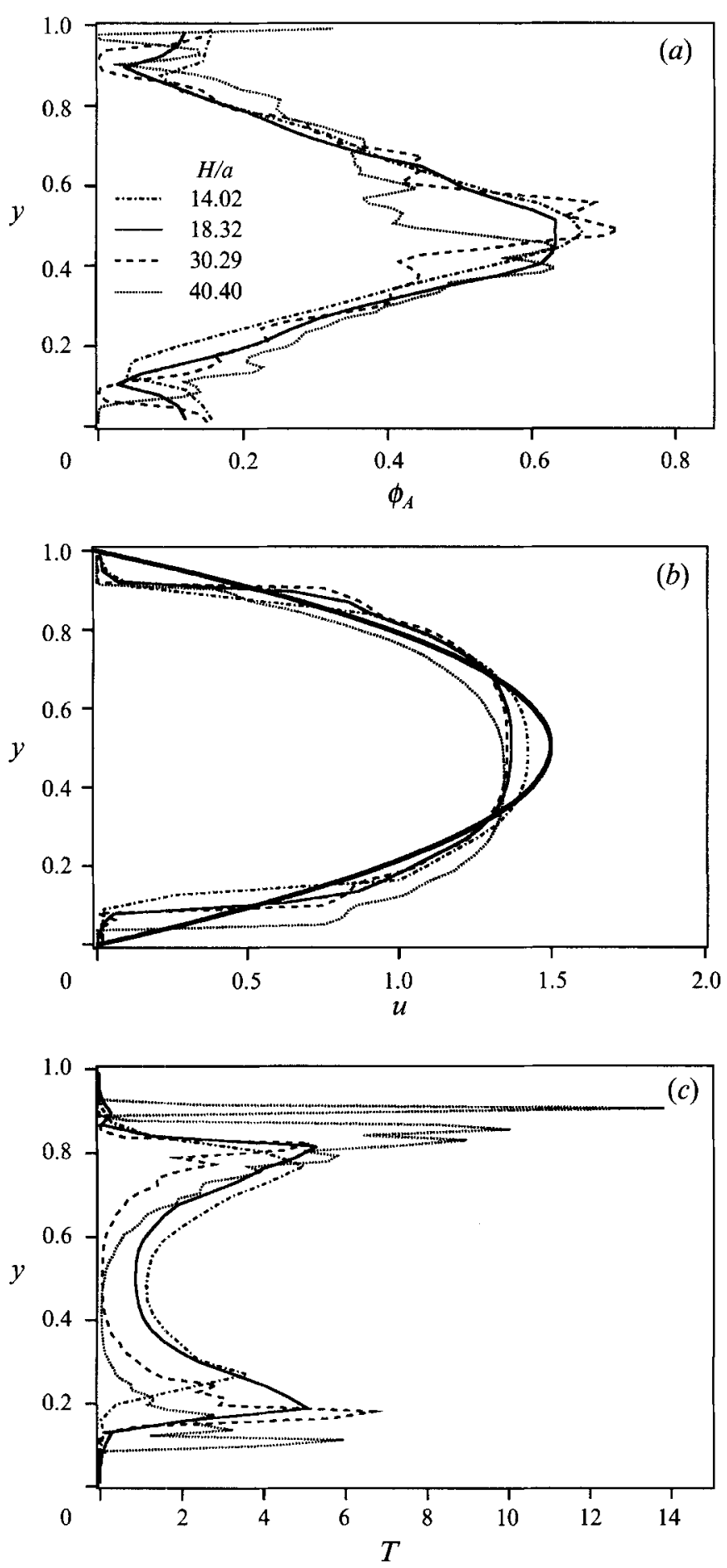

FIGURE 8 . The steady-state concentration $(a)$, velocity $(b)$ and suspension temperature $(c)$ profiles for the simulations with $H / a=14.02,18.32,30.29$ and 40.40 and $\phi_{A}^{b}=0.3$ (simulations $\mathrm{E}, \mathrm{F}, \mathrm{G}$ and $\mathrm{H}$ ). The dark line in $(b)$ is the parabolic velocity profile for a Newtonian fluid. The interparticle force in all cases was such that $\tau=1000$ and $F_{0}=0.001$. 


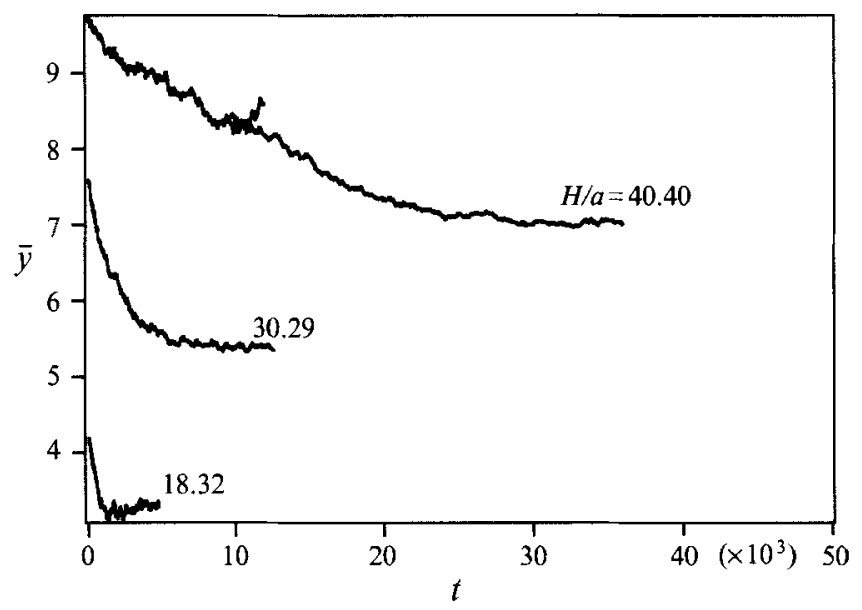

FIGURE 9. Comparison of the time trace of the average distance of particles from the centreline for three different channel widths at $\phi_{A}^{b}=0.4$.

\begin{tabular}{|c|c|c|c|c|}
\hline$\Delta \bar{y} / H$ & $H / a=$ & 40.40 & 30.29 & 18.32 \\
\hline 0.0125 & & 0.031 & 0.011 & 0.039 \\
\hline 0.025 & & 0.096 & 0.024 & 0.075 \\
\hline 0.03 & & 0.181 & 0.050 & 0.107 \\
\hline 0.050 & & 0.240 & 0.086 & 0.192 \\
\hline
\end{tabular}

TABLE 2. The elapsed time, scaled by $(H / a)^{3}$, at different migration distances, $\Delta \bar{y}$, for the three cases depicted in figure 9 . Note that this scaling normalizes the disparate migration timescales for the three channel widths to the same order of magnitude.

\subsection{Effect of the channel width}

We next explore the influence of the channel width in figures 7 and 8 , which present comparisons of the results for four channel sizes at average areal fractions of 0.4 and 0.3 respectively. With increasing $H / a$, the concentration at the centre rises slightly, the velocity profile becomes more blunted and there is a small drop in the velocity at the centreline. While the effect of $H / a$ on the concentration and velocity fields is quite weak, the temperature appears to be strongly influenced by it, decreasing uniformly with increasing $H / a$ except in the layers adjacent to the walls. Another point to be noted from figures $7(c)$ and $8(c)$ is that the thickness of the layers within which the temperature falls from its maximum to zero at the walls decreases with increasing $H / a$. This point assumes importance in the discussion of the models in $\S 6$. For the two widest channels in figure 7 , the concentration at the centre is close to maximum packing and the temperature is very small. This has therefore led to the formation of a relatively stable structure among the particles at the centre, which does not disappear even when averaged over a long period of time because the structure is rarely disturbed. The temperature in the smallest channel is considerably higher and there is hence no persistent structure in this case. The particle densities at the channel centre are somewhat lower when the bulk concentration is reduced to 0.3 (figure 8). Here, a skewed particle distribution for the case of $H / a=40.40$ (simulation D) has caused the particle density, velocity and temperature to be weighted towards one wall. While this is most likely a statistical fluctuation, the time required to smooth out these variations 

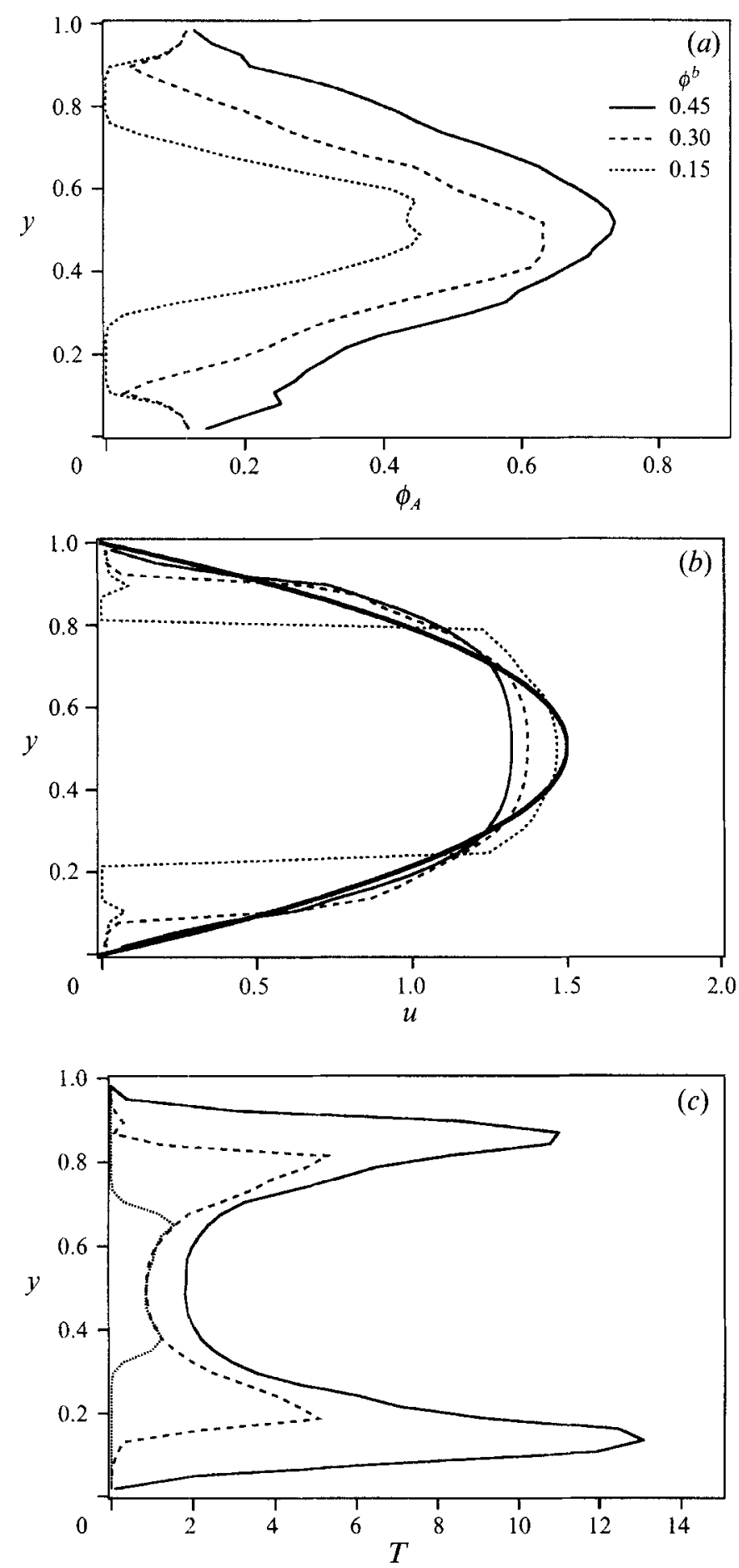

FIGURE 10. The steady-state concentration $(a)$, velocity $(b)$ and suspension temperature $(c)$ profiles for the simulations with $\phi_{A}^{b}=0.15,0.30$ and 0.45 and $H / a=18.32$ (simulations I, F and J). The dark line in $(b)$ is the parabolic velocity profile for a Newtonian fluid. The inter-particle force in all cases was such that $\tau=1000$ and $F_{0}=0.001$. 

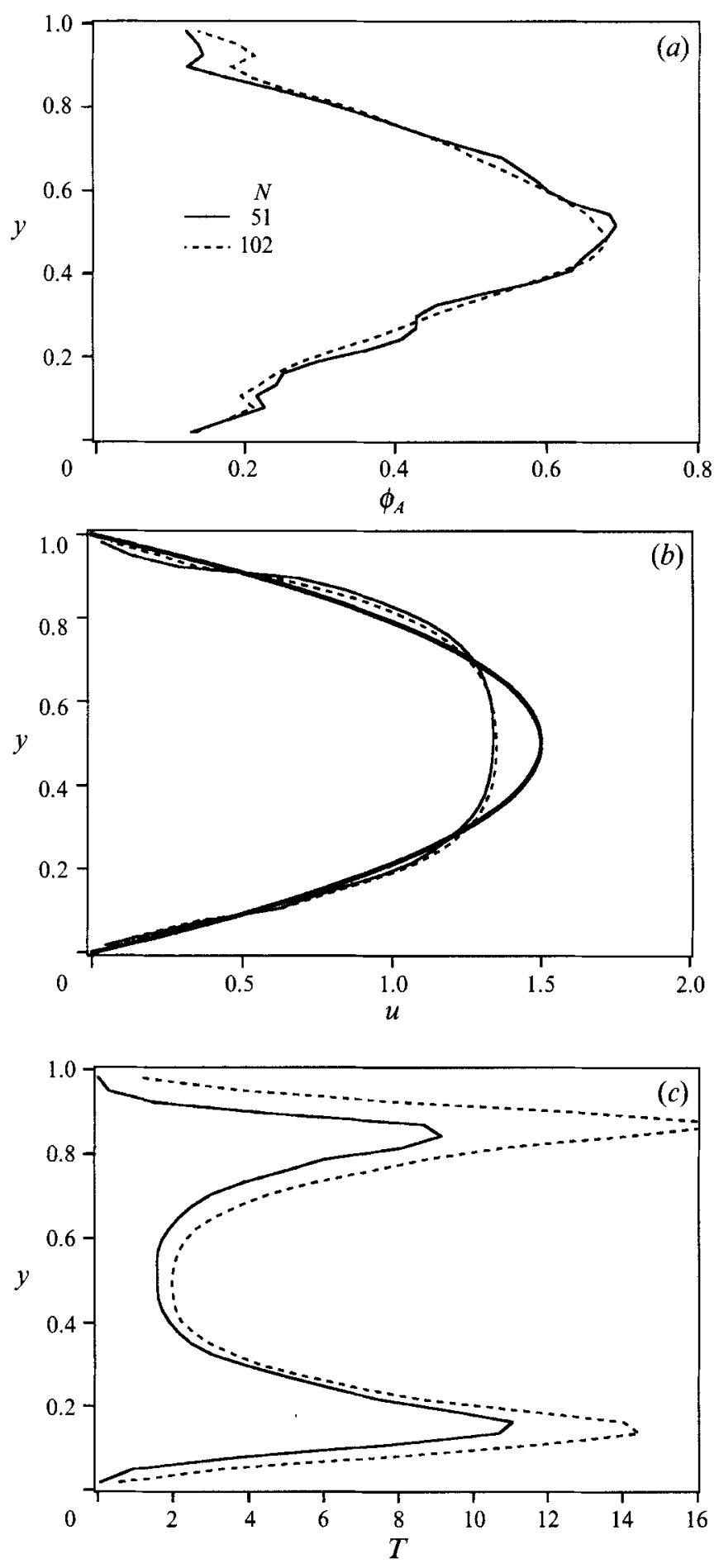

FIGURE 11. Comparison of the steady-state concentration (a), velocity $(b)$ and suspension temperature profiles $(c)$ for simulations B3 and B5, for which the number of particles in the unit cell were 51 and 102 , respectively. The bulk areal fraction in both cases in 0.4 and the channel width is 18.32 . The dark line in $(b)$ is the parabolic velocity profile for a Newtonian fluid. 
is large; ideally, simulations with many initial configurations should be conducted to derive proper averages, but this is not possible owing to the large computational costs. That aside, the influence of $H / a$ on the flow variables is the same as in the case of the larger bulk density in figure 7 .

The time trace of $\bar{y}$ for three different channel sizes at a bulk areal fraction of 0.4 are displayed in figure 9. The disparate timescales for achieving steady state in the three cases is quite clear. As a test of the $(H / a)^{3}$ scaling for the migration time that was predicted in $\S 2$, we have tabulated in table 2 the rescaled time $t(H / a)^{-3}$ against $\Delta \bar{y}$, the average distance traversed by the particles in time $t$, for the cases depicted in figure 9 . With this scaling, the variation in the migration time for the three different channel widths is substantially reduced, leading to a qualitative verification of the scaling law.

\subsection{Effect of concentration}

The effect of the bulk particle concentration on the system was studied by performing simulations $\mathrm{J}, \mathrm{F}$ and $\mathrm{I}$ at areal fractions of $0.45,0.3$ and 0.15 respectively, and a constant channel width of 18.32 particle radii. The results for the three concentrations are shown together in figure 10. Substantial segregation of particles even at the lowest concentration is apparent from the density plot. With increasing $\phi_{A}^{b}$, the areal fraction at the centre increases and the velocity at the centre decreases. At the lowest bulk concentration $\left(\phi_{A}^{b}=0.15\right)$, the central core is separated from the walls by a region of clear fluid. Hence, the particle velocity and temperature in this region are zero and it is as though a plug of particles is moving through the channel. The temperature is also strongly influenced by $\phi_{A}^{b}$, particularly near the walls where the shear rate is large.

\subsection{Effect of simulation size}

In order to test that the number of particles in the simulations was large enough so that the results were independent of the size of the unit cell, we have compared the steadystate results from simulations B3 and B5 in figure 11. The latter had twice as many particles as the former (i.e. the unit cell was twice as long), but the bulk concentration and channel width were left unchanged. The concentration and velocity fields in the two cases are almost identical. The temperature near the walls is higher for the larger unit cell, ostensibly because the number of particles trapped near the walls is greater.

\section{Comparison with earlier work}

We next compare the results of this work with those of earlier experimental investigations. Specifically, comparisons are made with the data of Karnis et al. (1966) and Koh et al. (1994). We emphasize here that these comparisons are mainly qualitative because the movement of particles in this work was restricted to two dimensions while the experiments, of course, imposed no such restriction.

In their experiments on pressure-driven flow of suspensions through tubes, Karnis et al. observed blunting of the particle velocity at the centre, but not discernible inhomogeneity in the concentration. Figure 12 displays their velocity data $\uparrow$ for a bulk volume fraction of 0.30 and a tube diameter of 35.71 particle radii, along with the velocity profile from simulation D. Karnis et al. characterize the blunting of the velocity field by the core radius, $r_{c}$, up to which the velocity is not measurably different from that at the centre - for this experiment they report a value of 0.38 for $r_{c} / R$. The

$\dagger$ Since they report particle velocities normalized by that at the axis, the ratio of the particle velocity to that of a Newtonian fluid (at the same flow rate) at the axis is needed to make this comparison. This ratio is estimated to be roughly 0.75 from their table 1 . 


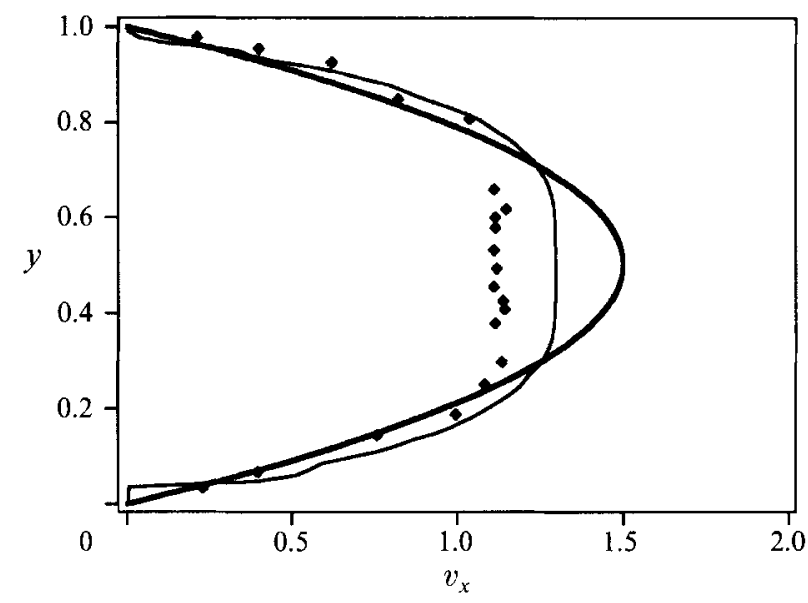

FIGURE 12. Comparison of the velocity profile for simulation D, $\phi_{A}^{b}=0.4$ and $H / a=40.40$ (with the data reported by Karnis et al. for $\phi^{b}=0.30$ and $D / a=35.71(\diamond)$. The dark line is the parabolic velocity profile for a Newtonian fluid.

present work shows less blunting in the velocity profile and the velocity within the core is higher. For a suspension of volume fraction 0.14 , they report that the velocity profile showed no deviation from the parabolic profile of a Newtonian fluid. In contrast, we observe appreciable deviation from the parabolic profile even for a volume fraction of 0.10 (areal fraction 0.15 ), as shown in figure $10(b)$. These discrepancies are quite probably a consequence of their measurements being made well within the development length of the tube. While the measurements of Karnis et al. were made less than 50 diameters from the entrance of the tube, the transition length estimated from (4) is $L / H \sim 1275$.

We next compare the velocity and density results obtained from the simulations with the data of Koh et al. Though they have reported data for a range of $H / a$ and $\phi^{b}$, only three of their experiments were for conditions close enough to the simulations of this work. These are for bulk volume fractions of $0.1,0.2$ and 0.3 , respectively, and at $H / a=17.54$; the corresponding simulations in the present work are I, F and $\mathrm{J}$ respectively, which are for the same concentrations and at $H / a=18.32$. Figure 13 shows the comparisons, with the lines representing the results of this work and the symbols representing the data of Koh et al. Note that the particle concentration is now given in terms of the volume fraction, with $\phi^{b}=2 / 3 \phi_{A}^{b}$. While the concentration profiles of Koh et al. and the present work are in good agreement for $\phi^{b}=0.2$, their measurements indicate a flatter concentration field for a bulk density of 0.1 and a more sharply segregated distribution for a bulk density of 0.3 . The difference between the two investigations for the largest bulk density is most likely because the maximum packing density for monolayers is smaller than when there is unrestricted movement in all three dimensions - they are roughly 0.55 and 0.63 (in volume fraction) respectively. With this difference taken into account, the concentration fields are in much closer agreement for $\phi^{b}=0.3$. At the smallest bulk density, however, (4) predicts that their measurements were not at steady state, leaving the migration incomplete and hence a flatter concentration profile than that of simulation I.

The velocity measurements of Koh et al. are substantially lower than what is observed in the simulations of this work. Such low particle velocities imply that the particles move much slower than the fluid around them, i.e. there is a sizeable phase slip. This is rather unusual, especially if the particles are freely suspended in the fluid 

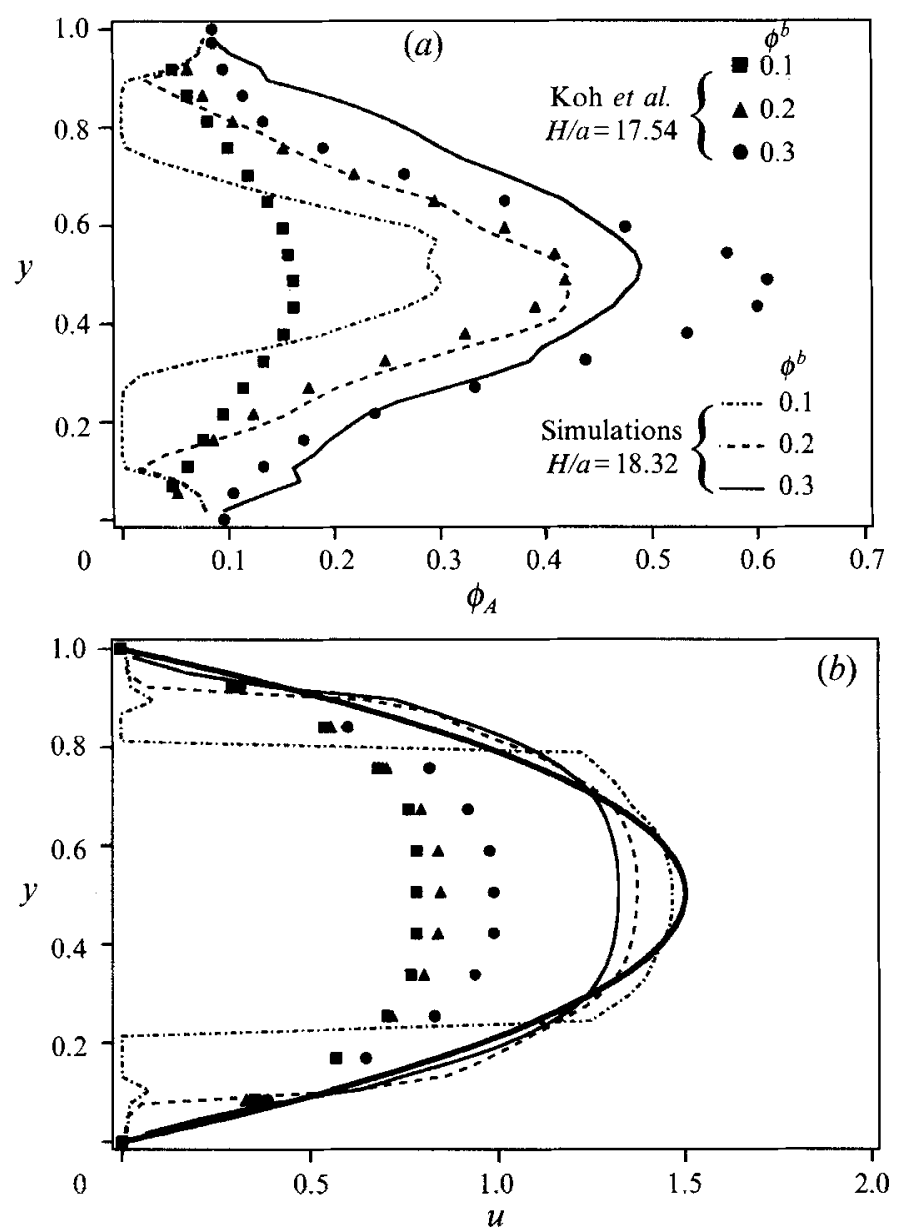

FIGURE 13. Comparison of the concentration $(a)$ and velocity $(b)$ profiles from this work with the results of Koh et al. (1994). Note that the concentrations are now given in terms of volume fractions. The dark line in $(b)$ is the parabolic velocity profile for a Newtonian fluid.

without any external forces acting on them. Indeed, the average particle velocities listed in table 1 for all the simulations are all very close to unity, indicating that there is almost no phase slip between the particles and fluid. The theoretical analysis in $\S 6.2$ also supports this conclusion. The particle flux $Q_{p}$ listed in column 10 of table 1 is defined as

$$
Q_{p}=\int \phi u \mathrm{~d} y / \phi^{b} \int\langle u\rangle \mathrm{d} y ;
$$

it is equal to unity when the particles are uniformly dispersed in the fluid and translate with the same velocity as the fluid. While Koh et al. report the particle flux to be less than unity for the cases shown here, the value of $Q_{p}$ is greater than unity for all the simulations in this work. We may therefore speculate that their velocity measurements are either inaccurate or there were conditions at the entry, exit or within the channel that hindered the movement of particles.

A final point to be made regarding the experiments of Koh et al. is that they did not measure the bulk concentration of particles inside the channel, but only in the reservoir upstream. Their measurements of the point densities in the channel were scaled so that the bulk density inside the channel equals that in the reservoir; the condition 
$\phi^{b}=\int \phi \mathrm{d} y$ was used to calibrate the density measurements. The method used by Koh et al. is therefore incapable of determining the actual bulk density inside the channel and, if the particles were indeed hindered from movement, it is possible that the concentration in the channel may have differed from the reported values.

Sinton \& Chow (1991) have reported velocity measurements and NMR intensity plots that qualitatively show the distribution of particles in pressure-driven tube flow. However, all their experiments were for $H / a>200$ and hence no appropriate comparison with their results is possible. Moreover, they too failed to detect any particle migration towards the tube axis; in fact, their intensity plots indicate a greater concentration of particles at the periphery. Though this is most likely an artifact of flow-induced relaxation effects in their NMR imaging technique (as they indicate), their measurements were also made at a distance from the tube entry which is far smaller than that required for reaching steady state as remarked in $\$ 2$.

\section{Macroscopic models for suspension flow}

\subsection{Diffusive flux model}

As mentioned in $\$ 1$, Leighton \& Acrivos (1987 $b$ ) proposed a diffusion model to explain their observations of long-time decrease and short-time increase in the effective viscosity of a suspension in a Couette viscometer. This model was used later by Phillips et al. (1992) to predict concentration inhomogeneity in the pressure-driven flow in a tube and in wide-gap Couette flow. In their model the net flux of particles consists of two contributions: a diffusive flux driven by a gradient in the shear rate and diffusion due to a gradient in the concentration (for which the diffusivity is proportional to the local shear rate, $\dot{\gamma}$ ). In their notation, the diffusion equation for the particles is

$$
\frac{\partial \phi}{\partial t}=\frac{\partial}{\partial y}\left[K_{c} a^{2} \phi \frac{\partial}{\partial y}(\dot{\gamma} \phi)+K_{\eta} a^{2} \dot{\gamma} \phi^{2} \frac{1}{\eta_{s}} \frac{\mathrm{d} \eta_{s}}{\mathrm{~d} \phi} \frac{\partial \phi}{\partial y}\right],
$$

where $K_{c}$ and $K_{\eta}$ are proportionality constants, and $\eta_{s}$ is the relative viscosity of the suspension. For the case of pressure-driven flow in a channel, the net flux across the channel is equal to zero at steady state and (20) reduces to

$$
\frac{\dot{\gamma} \phi}{\dot{\gamma}^{w} \phi^{w}}=\left(\frac{\eta_{s}^{w}}{\eta_{s}}\right)^{K_{\eta /} / K_{c}}
$$

where the superscript $w$ denotes properties at the walls. The shear rate is determined from the momentum equation for the suspension, which is treated as a generalized Newtonian fluid:

$$
\eta \eta_{s}(\phi) \dot{\gamma}=G
$$

where $G$ is the applied pressure gradient.

From experimental data on Couette flows, Phillips et al. found that $K_{c} / K_{\eta} \approx 0.66$; with the approximation that $1.82\left(1-K_{\eta} / K_{c}\right) \approx-1$ together with Kreiger's formula for the effective viscosity,

$$
\eta_{s}=\left(1-\phi / \phi_{m}\right)^{-1.82} \text {, }
$$

where $\eta$ and $\phi_{m}$ are the viscosity of the suspending fluid and the maximum packing volume fraction respectively, they derived

$$
\phi=\frac{\phi_{m}}{1+C y} .
$$


The constant $C=\left(\phi_{m}-\phi^{w}\right) / \phi^{w}$ is determined by the condition on the average particle concentration,

$$
\phi^{b}=\int_{0}^{1} \phi \mathrm{d} y=\frac{\phi_{m}}{C} \log (1+C) .
$$

The velocity profile is then obtained by solving the momentum equation,

$$
\dot{\gamma}=\frac{G}{\eta_{0}}\left(\frac{C y}{1+C y}\right)^{1.82} .
$$

The predictions of this model for channel flow at $\phi_{A}^{b}=0.45$ are shown in figure 14 . The model does indeed predict that particles migrate towards the centre, resulting in a velocity profile that is in reasonable agreement with simulation. However, since the shear rate at the centreline is zero by symmetry, the driving force for the diffusive flux in (20) vanishes and the concentration at the centre must therefore equal $\phi_{m}$. With the choice of the ratio $K_{c} / K_{\eta}$ and the form of the relative viscosity in (23), the concentration profile exhibits a sharp cusp at the centre. Furthermore, there is no dependence on the ratio $H / a$ in this model.

\subsection{Suspension balance model}

We now outline an alternative approach in which there is no diffusion, but rather mass, momentum and energy balances are written for the particulate phase (and the entire suspension) in a manner analogous to molecular or atomic systems. These balances are then solved simultaneously for the concentration, bulk velocity and suspension temperature. It will be seen that the macroscopic statement that there is no pressure variation perpendicular to the direction of mean motion will give an equation to predict particle migration and the concentration variations seen in inhomogeneous flows.

The basic idea that suggests such a model might be appropriate is that when one examines the governing equations of particle motion (cf. (11)) one notices that there is no explicit reference to the fluid (although there is to the 'bulk' material as explained below). The particles evolve according to Newton's laws of motion just as molecules, the difference is that the forces acting on them are given by rather complex configuration-dependent expressions. In other words, the fluid in this framework only determines the nature of interactions between particles, and once these interactions are known, no explicit reference to the fluid is necessary. From a statistical mechanics perspective there are no degrees of freedom associated with the fluid. (This is not true, of course, at finite Reynolds numbers where the degrees of freedom of the fluid enter explicitly.) It appears therefore that a macroscopic description of the particles as a continuum should be possible, just as in a molecular system.

One may obtain the so-called hydrodynamic equations from Newton's laws of motion for molecular systems by a process of averaging as first proposed by Irving \& Kirkwood (1950). Doing so, one arrives at the usual conservation laws of continuum mechanics: mass, momentum and energy, along with a microscopic interpretation of the stress tensor, energy flux, etc. Since the hydrodynamically interacting particles also evolve according to Newton's laws, we may derive the continuum equations of motion for the particles in a procedure analogous to that of molecular systems. Just as there is no diffusion in the conservation of mass for a molecular system, there is no diffusion in this description either. There can be, however, self-diffusion of a marked particle as discussed in the next section. 
The macroscopic equations can be obtained simply by averaging the equation of conservation of mass

$$
\frac{\partial \rho}{\partial t}+\nabla \cdot \rho \boldsymbol{u}=0
$$

and Cauchy's equation of motion

$$
\rho \frac{\mathrm{D} u}{\mathrm{D} t}=\boldsymbol{b}+\boldsymbol{\nabla} \cdot \boldsymbol{\sigma},
$$

which apply at any material point - in particles or fluid. Here, $b$ represents the body force per unit volume and/or non-hydrodynamic interparticle forces, and $\sigma$ is the stress tensor. Using the phase indicator function

$$
\chi= \begin{cases}1 & \text { in particles } \\ 0 & \text { in fluid }\end{cases}
$$

it is straightforward to average (27) and (28) over the particles (Drew \& Lahey 1993), which is nothing more than the continuum mechanics version of the Irving-Kirkwood procedure for point particles. The conservation equations for particle mass and momentum are simply

$$
\begin{gathered}
\frac{\partial \phi}{\partial t}+\nabla \cdot \phi\langle\boldsymbol{u}\rangle_{p}=0, \\
\rho_{p} \phi \frac{\mathrm{D}_{p}\langle\boldsymbol{u}\rangle_{p}}{\mathrm{D} t}=\langle\boldsymbol{b}\rangle_{p}+\langle\boldsymbol{F}\rangle_{p}+\boldsymbol{\nabla} \cdot\langle\boldsymbol{\Sigma}\rangle_{p},
\end{gathered}
$$

where we have assumed that the density of a particle, $\rho_{p}$, is constant within each particle. The average particle velocity, $\phi\langle\boldsymbol{u}\rangle_{p} \equiv\langle\chi \boldsymbol{u}\rangle$, where \langle\rangle denotes an average over all material, is equivalently the number average, volume average or mass average for constant $\rho_{p}$. The substantial derivative is that following the average particle motion

$$
\frac{\mathrm{D}_{p}}{\mathrm{D} t} \equiv \frac{\partial}{\partial t}+\langle\boldsymbol{u}\rangle_{p} \cdot \boldsymbol{\nabla}
$$

The body force per unit volume exerted on the particles $\langle\boldsymbol{b}\rangle_{p}$ is the product of the number density of particles, $n$, and the average body force per particle $\langle\boldsymbol{b}\rangle_{p} \equiv$ $n(1 / N) \sum_{\alpha=1}^{N} b^{\alpha}$, the sum being over all $N$ particles in an averaging volume.

The average stress of the particles, $\langle\Sigma\rangle_{p}$, is given by

$$
\langle\boldsymbol{\Sigma}\rangle_{p}=\langle\boldsymbol{\sigma}\rangle_{p}-\langle\boldsymbol{x} \boldsymbol{b}\rangle_{p}-\rho_{p}\left\langle\boldsymbol{u}^{\prime} \boldsymbol{u}^{\prime}\right\rangle_{p} .
$$

The last term on the right-hand side of (31) is the usual Reynolds stress term. The first moment of the non-hydrodynamic interparticle forces, $\langle\boldsymbol{x} \boldsymbol{b}\rangle_{p}$, is the stress that arises in molecular or colloidal systems from interparticle forces. And, $\langle\sigma\rangle_{p}$ is simply the average stress in the particles. Equating the ensemble and volume averages, these two terms become

$$
\langle\boldsymbol{\sigma}\rangle_{p}-\langle\boldsymbol{x} \boldsymbol{b}\rangle_{p}=\frac{1}{V} \sum_{\alpha=1}^{N} \int_{V_{\alpha}}(\boldsymbol{\sigma}-\boldsymbol{x} \boldsymbol{b}) \mathrm{d} \boldsymbol{V},
$$

and from Cauchy's equation and the divergence theorem

$$
\int_{V_{\alpha}}(\boldsymbol{\sigma}-\boldsymbol{x} \boldsymbol{b}) \mathrm{d} V=\int_{S_{\alpha}} r \boldsymbol{\sigma} \cdot \boldsymbol{n} \mathrm{d} S-\rho_{p} \int_{V_{\alpha}} r \boldsymbol{a} \mathrm{d} V-\boldsymbol{x}^{\alpha} \boldsymbol{b}^{\alpha} V_{\alpha},
$$


where $\boldsymbol{r}=\boldsymbol{x}-\boldsymbol{x}^{\alpha}$, with $\boldsymbol{x}^{\alpha}$ the location vector of the centre of particle $\alpha$, and $\boldsymbol{a}=\mathrm{D} \boldsymbol{u} / \mathrm{D} \boldsymbol{t}$ is the acceleration of the particle. We have also assumed that the particle force $b^{\alpha}$ is constant within the particle.

The surface integral in (32) is just the first moment of the hydrodynamic force density integrated over the surface of the rigid particle. The symmetric part is the stresslet (Batchelor 1970):

$$
\boldsymbol{S}^{\alpha} \equiv \int_{S_{\alpha}} \frac{1}{2}[\boldsymbol{r} \boldsymbol{\sigma} \cdot \boldsymbol{n}+\boldsymbol{\sigma} \cdot \boldsymbol{n} \boldsymbol{r}] \mathrm{d} S,
$$

and note that the stresslet as defined in (33) is not traceless. Indeed, the trace will be an important element in the macroscopic modelling.

The antisymmetric part of the first moment gives the hydrodynamic torque exerted on the particles, and, along with the antisymmetric part of the acceleration term $\int r a \mathrm{~d} V$, is equal to the total external torque $\boldsymbol{L}^{e x t}$ exerted on a particle. Thus, the particle stress becomes

$$
\langle\boldsymbol{\Sigma}\rangle_{p}=\langle\boldsymbol{S}\rangle_{p}+\frac{1}{2} \boldsymbol{\varepsilon}:\left\langle\boldsymbol{L}^{e x t}\right\rangle_{p}-\left\langle\boldsymbol{x}^{\alpha} \boldsymbol{b}^{\alpha}\right\rangle_{p}-\rho_{p} \frac{1}{2}\langle(\boldsymbol{r} \boldsymbol{a}+\boldsymbol{a r})\rangle_{p}-\rho_{p}\left\langle\boldsymbol{u}^{\prime} \boldsymbol{u}^{\prime}\right\rangle_{p} .
$$

It is informative to compare (34) for the particle stress to that for a molecular system. Both the interparticle force and Reynolds stress terms are present for molecular systems. The acceleration and torque contributions would also be present if the molecules were not considered to be points. The only new term is the hydrodynamic stresslet $\langle\boldsymbol{S}\rangle_{p}$; it has no counterpart in molecular systems. Note that in general there are inertial effects in the hydrodynamic stresslet through the dependence of $\sigma$ on the Reynolds number. The macroscopic particle stress tensor can be antisymmetric owing to both the external couple and to non-central interparticle forces (Bonnecaze \& Brady 1992).

Finally, in (30) $\langle\boldsymbol{F}\rangle_{p}$ is the average hydrodynamic force per unit volume exerted on the particles. This term arises naturally from the phase indicator function as $\langle\chi \nabla \cdot \sigma\rangle=$ $\nabla \cdot\langle\chi \sigma\rangle-\langle\sigma \cdot \nabla \chi\rangle$, and $\nabla \chi$ is a delta function on the surfaces of the particles with normal pointing into the particles.

The mass and momentum balances are valid for any material and for motion at any Reynolds number. What distinguishes one system from another is the form of the constitutive relations (for hydrodynamic drag force and stress, for example). We shall assume in what follows that the average body force is known. For our applications to low-Reynolds-number flows (and the restriction is only to low Reynolds numbers based on the particle size) the drag force is simply given by (cf. (6))

$$
\begin{aligned}
\langle\boldsymbol{F}\rangle_{p} & =-n\left\langle\boldsymbol{R}_{F U} \cdot(\boldsymbol{u}-\langle\boldsymbol{u}\rangle)\right\rangle_{p} \\
& \approx-n\left\langle\boldsymbol{R}_{F U}\right\rangle_{p} \cdot\left(\langle\boldsymbol{u}\rangle_{p}-\langle\boldsymbol{u}\rangle\right) \\
& \approx-6 \pi \eta \operatorname{anf}(\phi)^{-1}\left(\langle\boldsymbol{u}\rangle_{p}-\langle\boldsymbol{u}\rangle\right),
\end{aligned}
$$

where we have assumed an isotropic structure for $\boldsymbol{R}_{F U}$ and introduced the hindered settling function $f(\phi)$. In a gravitational settling experiment (30) and (35) will give $\langle\boldsymbol{u}\rangle_{p}-\langle\boldsymbol{u}\rangle=f(\phi) \boldsymbol{u}_{s}$, with $\boldsymbol{u}_{s}$ the Stokes settling velocity of an isolated particle. The hindered settling function has been measured experimentally and calculated by Stokesian Dynamics in homogeneous simulations (Phillips, Brady \& Bossis 1988).

Since our interest here is in low-particle-Reynolds-number flows, we shall discard the acceleration and Reynolds stress terms in the particle stress. The constitutive law for the stresslet and interparticle forces can be deduced from low-Reynolds-number hydrodynamics. For non-Brownian suspensions, the response is viscous (Brady $1993 b$ ), and the constitutive law becomes

$$
\langle\Sigma\rangle_{p}=-\Pi \boldsymbol{I}+2 \eta \eta_{p}(\phi)\langle\boldsymbol{e}\rangle+\eta\langle\boldsymbol{\chi}\rangle,
$$


where $\eta_{p}(\phi)$ is the relative viscosity of the particle phase. From the definition of the stresslet, it is easy to see that in the dilute limit for spheres $\eta_{p} \sim \frac{5}{2} \phi$. The isotropic stress $\Pi$ is the particle pressure and shall be discussed more fully below.

Even for motion at low Reynolds numbers, high Péclet numbers and with no or very weak inter-particle forces, there may still be normal stress differences, $\langle\boldsymbol{\chi}\rangle$, in viscous suspension flows (Gadala-Maria \& Acrivos 1980; Brady \& Bossis 1985; Phung 1993). For viscous suspensions the normal stresses are linear in the rate of strain

$$
\langle\boldsymbol{\chi}\rangle=\boldsymbol{A} \cdot\langle\boldsymbol{e}\rangle,
$$

where the fourth-order tensor $\boldsymbol{A}$ must change sign upon flow reversal, because the normal stress differences do not, but is otherwise independent of the magnitude of $\langle\boldsymbol{e}\rangle$. Little is known about the form of $\boldsymbol{A}$ other than that both the first and second normal stresses differences exist and are of comparable magnitude (Phung 1993). In rectilinear flow, such as flow in a channel or tube, the normal stress differences are not important. However, in flows with curvilinear streamlines, such as the torsional flow between rotating disks, these normal stress differences are important and may be responsible for the lack of migration in these flows as will be shown below.

The notion of a particle pressure has been controversial, especially for motion at low Reynolds numbers. However, a well-defined calculable pressure exists as simply the trace of the particle stress

$$
\begin{aligned}
3 \Pi & =-\boldsymbol{I}:\langle\boldsymbol{\Sigma}\rangle_{p} \\
& =-\boldsymbol{I}:\langle\boldsymbol{S}\rangle_{p}+\left\langle\boldsymbol{x}^{\alpha} \cdot \boldsymbol{b}^{\alpha}\right\rangle_{p}+\rho_{p}\langle\boldsymbol{r} \cdot \boldsymbol{a}\rangle_{p}+\rho_{p}\left\langle u^{\prime 2}\right\rangle_{p} .
\end{aligned}
$$

The interparticle force and the kinetic or Reynolds stress contributions are the same as in molecular systems, as would be the acceleration term for finite-size particles. The hydrodynamic contribution from the trace of the stresslet is not present in molecular systems and can be dominant at low Reynolds numbers. This term has not received attention in the past and has only recently been determined for low-Reynolds-number flows (Jeffrey, Morris \& Brady 1993). For a suspension of Brownian particles this hydrodynamic pressure can be shown to be the mechanical origin of the osmotic pressure of colloidal dispersions (Brady 1993a), and we have chosen the symbol $\Pi$ because of this correspondence.

The pressure is just another viscous stress, and therefore must scale linearly with the shear rate. However, upon flow reversal neither the pressure nor the normal stress differences change sign, unlike the shear stress, and therefore the scalar pressure can be represented as

$$
\Pi=\Pi_{0}+\eta \bar{p}(\phi) \dot{\gamma}
$$

where the rate of strain $\dot{\gamma}$ is defined as

$$
\dot{\gamma} \equiv(\langle e\rangle:\langle e\rangle)^{1 / 2} .
$$

In (39) $\Pi_{0}$ is a constant and $\bar{p}(\phi)$ is a non-dimensional and monotonically increasing function of the volume fraction. This form for the pressure is sufficient to understand and explain the phenomena of shear-induced particle migration and concentration variations in inhomogeneous flow, but it does require modification when the shear rate vanishes as discussed below.

The form of the constitutive laws for the drag force and particle stress involve the suspension average fields $\langle\boldsymbol{u}\rangle$ and $\langle\boldsymbol{e}\rangle$. These fields appear in the macroscopic balance equations because they explicitly determine the microscopic evolution of the particles. We shall need therefore balance laws for these fields in order to close the governing 
equations. One can, perhaps, appreciate the necessity for this by considering an electrostatic analogue. The motion of individual dipolar molecules depends on the local electric field they experience. The average dipole moment over a small representative volume gives the dielectric constant of the medium at that point. If a macroscopic electrostatic potential difference is now applied across the sample, the electric field experienced locally will depend on the local dielectric constant. Thus the motion of the individual dipole particles is coupled to their average through the dielectric response of the material. The analogous situation arises in suspension problems and the particle balances are coupled to the suspension balances directly from the microscale (cf. (11)).

The balance equations for the entire material or suspension as a whole can be obtained from the conservation of mass and Cauchy's equation of motion by averaging without the phase indicator function. For an incompressible material

$$
\begin{gathered}
\boldsymbol{\nabla} \cdot\langle\boldsymbol{u}\rangle=0, \\
\frac{\mathrm{D}\langle\rho \boldsymbol{u}\rangle}{\mathrm{D} t}=\langle\boldsymbol{b}\rangle+\boldsymbol{\nabla} \cdot\langle\boldsymbol{\Sigma}\rangle .
\end{gathered}
$$

The convective derivative is now with the suspension average velocity, and the suspension stress is of the same form as (31) with the averages now over the entire material. The Reynolds stress is now, of course, $\left\langle(\rho \boldsymbol{u})^{\prime} \boldsymbol{u}^{\prime}\right\rangle$. The low-Reynolds-number constitutive law for the stress is

$$
\begin{aligned}
\langle\Sigma\rangle & =-\langle p\rangle_{f} \boldsymbol{I}+2 \eta\langle\boldsymbol{e}\rangle+\langle\Sigma\rangle_{p} \\
& =-\langle p\rangle \boldsymbol{I}+2 \eta \eta_{s}(\phi)\langle\boldsymbol{e}\rangle+\eta\langle\chi\rangle,
\end{aligned}
$$

where

$$
\begin{gathered}
\langle p\rangle=\langle p\rangle_{f}+\Pi, \\
\eta_{s}(\phi)=1+\eta_{p}(\phi),
\end{gathered}
$$

and $\langle p\rangle_{f}$ is the average pressure in the fluid.

In this suspension balance model there is no diffusive motion, but there is particle migration from regions of high shear rate to low. To see this, consider a steady fully developed flow in the $x$-direction with variation of properties in the $y$-direction. The $x$ momentum balance will determine the velocity profile, and the $y$-momentum balance becomes simply

$$
\frac{\partial \Pi}{\partial y}=\frac{\partial}{\partial y}(\eta \bar{p}(\phi) \dot{\gamma})=0 .
$$

(Technically, if normal stress differences are present one should write $\left\langle\Sigma_{y y}\right\rangle_{p}$ and not just the pressure $\Pi$, but since the normal stresses scale the same, the conclusions are unchanged.) Since the shear rate varies in the $y$-direction, the concentration must also vary in such a way as to keep the pressure constant. Where the shear rate is low the concentration is high and vice versa - the particles migrate to the centre. This is all that is needed to explain the phenomenon of shear-induced particle migration. Indeed, the same arguments will apply to any multiphase flow and can explain, for example, why particles in high-Reynolds-number flow in a channel migrate to the walls. In that situation the pressure scales inertially as $\rho u^{\prime 2}$, and the velocity fluctuations are largest in the centre where the mean velocity is highest; hence the particles migrate from the centre to the walls.

It is also straightforward to show that the diffusive flux model is contained within this suspension balance model. Consider again a unidirectional fully developed shear 
flow, with the flow in the $x$-direction and all variations in the $y$-direction. The equations for conservation of particles and $y$-momentum are then

$$
\begin{aligned}
\frac{\partial \phi}{\partial t}+\frac{\partial}{\partial y}\left(\phi\langle v\rangle_{p}\right) & =0, \\
\frac{\partial}{\partial y}(\bar{p} \dot{\gamma}) & =-\frac{9}{2} a^{-2} \phi f^{-1}\langle v\rangle_{p},
\end{aligned}
$$

where $v$ is the $y$-component of velocity. Substituting for $\phi\langle v\rangle_{p}$ into the particle conservation equation, we obtain

$$
\frac{\partial \phi}{\partial t}-\frac{\partial}{\partial y} \frac{2}{9} a^{2}\left(f \bar{p}^{\prime} \dot{\gamma} \frac{\partial \phi}{\partial y}+f \bar{p} \frac{\partial \dot{\gamma}}{\partial y}\right)=0
$$

which is a 'diffusion' equation for the particles and is of the same form as that proposed by Phillips et al. (1992, equation (20)).

As constructed so far the suspension balance model is very similar to the diffusive flux model. It has the same weakness in regions of zero shear rate - the concentration must approach maximum packing so that the pressure remains constant. (The pressure function $\bar{p}$ diverges as maximum packing is approached.) However, the suspension balance model can explain the lack of migration in torsional flow in the recent experiments of Chow et al. (1994). In torsional flow, the velocity is in the $\theta$-direction, grows linearly with the radius $r$ and has a gradient in the $z$-direction: $\langle\boldsymbol{u}\rangle=\operatorname{rh}(z) \hat{\boldsymbol{\theta}}$. The radial momentum balance can be written as

$$
\frac{\partial\left(\Pi-\left\langle\chi_{r r}\right\rangle_{p}\right)}{\partial r}=\frac{\left\langle\chi_{r r}\right\rangle_{p}-\left\langle\chi_{\theta \theta}\right\rangle_{p}}{r} .
$$

Because of the normal stress differences, the variation of the pressure (or more correctly the full $r$-normal stress) in the radial direction is not zero, and even though the shear rate grows with $r$ the concentration need not vary. In fact, $\langle\chi\rangle$ and $\Pi$ both grow linearly with $r$, so (48) implies a relation among $\bar{p}$ and the first and second normalstress difference functions, $\Psi_{1}$ and $\Psi_{2}$ (cf. Bird, Armstrong \& Hassager 1977) to prevent migration. Little is known about these functions, but the sign appears to be correct (Gadala-Maria \& Acrivos 1980; Brady \& Bossis 1985; Phung 1993). It should be noted that even with the same constitutive law for the stress, the diffusive flux model would necessarily predict radial migration because it is wedded to the concept of diffusion proportional to the shear rate.

To remove the other failing of the diffusive flux model in regions where the shear rate vanishes, we return to a consideration of the constitutive equation for the pressure and introduce the suspension temperature. As constructed both models are local-the stress, diffusivity, etc. are related to field variables at the same point. The finite size of the particles, however, implies that if variations occur on the scale of a particle then this local description breaks down. In particular, when the shear rate is small the finite size of a particle and its close proximity to neighbours at high $\phi$ will allow it to sample nearby regions where the shear rate is not zero, and therefore particle motion and stress will still be generated at a point where the macroscopic shear rate is zero. A non-local description will also naturally bring in the ratio of the particle size to the scale of the flow, $a / H$, which was observed to have an effect in the simulations.

A non-local expression for the stress would introduce a dependence on $\nabla \phi$ and $\boldsymbol{\nabla}\langle\boldsymbol{e}\rangle$. General considerations of symmetry show that there should be no terms linear 
in these gradients, and one must go to second order with all possible combinations of $\boldsymbol{\nabla} \phi \boldsymbol{\nabla} \phi, \boldsymbol{\nabla}\langle\boldsymbol{e}\rangle \boldsymbol{\nabla}\langle\boldsymbol{e}\rangle, \boldsymbol{\nabla} \phi \boldsymbol{\nabla}\langle\boldsymbol{e}\rangle, \boldsymbol{\nabla} \phi, \boldsymbol{\nabla} \nabla\langle\boldsymbol{e}\rangle$, etc. While such a theory could possibly be constructed, the number and form of the terms would introduce too many unknown phenomenological functions of $\phi$ to be modelled. There is an alternative way to capture the same physics that is much simpler and, more importantly, recognizes that the fluctuational motion of the particles - the suspension temperature - is an important element in determining the behaviour of concentrated suspensions.

To motivate the need for the temperature, note that for low-Reynolds-number flows the hydrodynamic pressure has contributions from the motion of the particles relative to the average and from the bulk straining motion:

$$
\boldsymbol{I}:\langle\boldsymbol{S}\rangle_{p}=\langle\boldsymbol{P} \cdot(\boldsymbol{u}-\langle\boldsymbol{u}\rangle)-\boldsymbol{Q}:\langle\boldsymbol{e}\rangle\rangle_{p},
$$

where $\boldsymbol{P}$ and $\boldsymbol{Q}$ are the contraction of $\boldsymbol{R}_{S U}$ and $\boldsymbol{R}_{S E}$ with $\boldsymbol{I}$, respectively. We argue that the pressure should be related to the fluctuational motion of the particles, i.e. the suspension temperature, and thus in place of (39) we write

$$
\Pi=\Pi_{0}+\eta a^{-1} p(\phi) T^{1 / 2},
$$

where $p(\phi)$ is a non-dimensional function of the volume fraction and $T=\left\langle\boldsymbol{u}^{\prime} \cdot \boldsymbol{u}^{\prime}\right\rangle_{p}$. This is of the same form as in a gas (or granular flow), where the pressure comes from the Reynolds stress term (and the interparticle forces in a dense gas) and is proportional to the temperature. In hydrodynamically driven motion at low Reynolds numbers, from (49) we see there is a term directly proportional to the first power of the particle velocity fluctuations, hence dimensionally proportional to the solvent viscosity, inversely proportional to the particle size and proportional to the square root of the temperature. In a homogeneous shear flow the temperature is proportional to the rate of strain squared, $\dot{\gamma}^{2}$, and thus writing the pressure as proportional to $T^{1 / 2}$ or to $\dot{\gamma}$ are entirely equivalent; only the non-dimensional proportionality function changes from $\bar{p}(\phi)$ to $p(\phi)$. In inhomogeneous flow, the temperature need not be proportional to the shear rate squared, need not be zero where the shear rate is zero, and (50) will incorporate the quadratic non-local terms needed.

Another argument that suggests that the temperature is appropriate comes from the considerations that relate the particle diffusivity to the velocity fluctuation autocorrelation function in $\S 2$ :

$$
D \equiv \int_{0}^{\infty}\left\langle u^{\prime}(0) \cdot u^{\prime}(t)\right\rangle_{p} \mathrm{~d} t \sim a T^{1 / 2}
$$

The particle diffusivity is fundamentally linked to the temperature, rather than the shear rate, as the model of Leighton \& Acrivos assumes. This change in perspective is important because the temperature can remain finite even though the shear rate is zero.

With the introduction of the suspension temperature as a fundamental variable determining the particle pressure, a balance law for the fluctuational motion is needed. Since particle motion at low Reynolds numbers is intimately tied to the motion of the suspension as a whole, it is simplest to arrive at a equation for the temperature by considering the mechanical energy balance for the entire material. Multiplying Cauchy's equation of motion by $\boldsymbol{u}$, averaging over the entire suspension and subtracting $\langle\boldsymbol{u}\rangle$ contracted with (41) gives (see also Batchelor 1970)

$$
\frac{\mathrm{D}\left\langle\frac{1}{2}\left(\rho u^{2}\right)^{\prime}\right\rangle}{\mathrm{D} t}=\left\langle\boldsymbol{b}^{\prime} \cdot \boldsymbol{u}^{\prime}\right\rangle+\langle\Sigma\rangle:\langle\boldsymbol{e}\rangle-\langle\dot{\Phi}\rangle-\nabla \cdot\langle\boldsymbol{q}\rangle
$$


The left-hand side is rate of accumulation of fluctuational energy, $\left\langle\boldsymbol{b}^{\prime} \cdot \boldsymbol{u}^{\prime}\right\rangle$ is the rate of working by body forces, $\langle\boldsymbol{\Sigma}\rangle:\langle\boldsymbol{e}\rangle$ is the rate of working by the bulk stress (and, for simplicity, we have assumed the bulk stress to be symmetric), $\langle\dot{\Phi}\rangle$ is average rate of dissipation of mechanical energy into heat, and the last term is the divergence of the 'heat' flux vector

$$
\langle\boldsymbol{q}\rangle \equiv-\left\langle\boldsymbol{\Sigma}^{\prime} \cdot \boldsymbol{u}^{\prime}\right\rangle
$$

The mechanical energy balance is of the same form as in a molecular system, and the dissipation can be expressed in terms of the particle temperature.

In low-Reynolds-number flows, the dissipation of energy in a linear flow due to the particles (there is also, of course, the dissipation due to the solvent, $\eta\langle\boldsymbol{e}\rangle:\langle\boldsymbol{e}\rangle$ ) is given by the generalized vector of velocities dotted into the generalized hydrodynamic force (Brady \& Bossis 1988)

$$
\dot{\Phi}=\left(\begin{array}{c}
\boldsymbol{u}-\langle\boldsymbol{u}\rangle \\
-\langle\boldsymbol{e}\rangle
\end{array}\right) \cdot\left(\begin{array}{ll}
\boldsymbol{R}_{F U} & \boldsymbol{R}_{F E} \\
\boldsymbol{R}_{S U} & \boldsymbol{R}_{S E}
\end{array}\right) \cdot\left(\begin{array}{c}
\boldsymbol{u}-\langle\boldsymbol{u}\rangle \\
-\langle\boldsymbol{e}\rangle
\end{array}\right) .
$$

Noting that $\boldsymbol{u}-\langle\boldsymbol{u}\rangle=\left(\boldsymbol{u}-\langle\boldsymbol{u}\rangle_{p}\right)+\left(\langle\boldsymbol{u}\rangle_{p}-\langle\boldsymbol{u}\rangle\right)$, we see that the average rate of dissipation will involve several distinct terms. There is the dissipation due to an affine motion with all particles moving with the mean velocity gradient: $\langle\boldsymbol{e}\rangle:\left\langle\boldsymbol{R}_{S E}\right\rangle_{p}:\langle\boldsymbol{e}\rangle$. There is a similar affine dissipation due to all particles moving relative to the mean suspension velocity, as will occur when there is phase slip due to a body force acting on the particles: $\left(\langle\boldsymbol{u}\rangle_{p}-\langle\boldsymbol{u}\rangle\right) \cdot\left\langle\boldsymbol{R}_{F U}\right\rangle_{p} .\left(\langle\boldsymbol{u}\rangle_{p}-\langle\boldsymbol{u}\rangle\right)$. And there will be the dissipation caused by the fluctuational motion of the particles about their mean: $\left\langle\boldsymbol{u}^{\prime} \cdot \boldsymbol{R}_{F U} \cdot \boldsymbol{u}^{\prime}\right\rangle_{p}$. There are also cross-terms coupling particle velocity fluctuations to the rate of strain and to the mean particle motion relative to the suspension average.

In steady homogeneous shear flow the difference between the macroscopic rate of working, $\langle\boldsymbol{\Sigma}\rangle:\langle\boldsymbol{e}\rangle$, and the dissipation of the affine motion is dissipated in the fluctuational motion and serves as a equation to determine the fluctuations. The fluctuational dissipation is proportional to the particle temperature. The importance of this splitting of the rate of working into affine and fluctuational motion can be appreciated by considering the shearing of a periodic lattice of particles. The particles will all move affinely and the dissipation due to viscous forces is quite large, but there is no fluctuational motion of the particles.

With this splitting of the dissipation, the fluctuational energy balance for the entire suspension can be written as

$$
c(\phi) \frac{\mathrm{D}\left\langle\frac{1}{2}\left(\rho u^{2}\right)^{\prime}\right\rangle}{\mathrm{D} t}=\beta(\phi)\left\langle\boldsymbol{b}^{\prime} \cdot \boldsymbol{u}^{\prime}\right\rangle+\langle\Sigma\rangle_{p}:\langle\boldsymbol{e}\rangle-\eta \alpha(\phi) a^{-2} T-\nabla \cdot\langle\boldsymbol{q}\rangle,
$$

where we have introduced the non-dimensional phenomenological coefficients, $\alpha(\phi)$ giving the temperature from shearing motion, $\beta(\phi) / \alpha(\phi)$ giving the temperature in sedimentation, and $c(\phi)$ representing the "heat capacity'. These latter two functions are needed because we have chosen to leave the actual rate of shear working unmodified, although only part of this goes into the fluctuational motion. (Note that the rate of shear working now involves the particle stress as the fluid contribution is part of the affine motion.) The volume-fraction-dependent functions, $\alpha, \beta$ and $c$ can all be determined in homogeneous experiments or Stokesian Dynamics simulations.

While the macroscopic equations are phenomenologically similar to those used to model the behaviour of dry granular flows, and this analogy has been put forward by Jenkins \& McTigue (1990), the origin of the velocity fluctuations is quite different from 
that in granular flows. (In dry granular flows dissipation occurs within particles due to inelastic collisions, and the rate of dissipation scales as the granular temperature to the $\frac{3}{2}$ power.) On dimensional grounds one can argue the forms of the constitutive laws, but to properly determine their behaviour requires careful consideration of the underlying physics. If one recalls that the Navier-Stokes equations apply to any atomic or molecular system quite independent of whether a gas or a liquid, only the coefficients of the viscosity and their dependence on molecular properties distinguish a gas from a liquid, then it is not at all surprising that the phenomenological form of the macroscopic equations for viscous suspension flows should be the same as for dry granular flows. The important distinction between molecular and macroscopic systems is that the temperature (at least at steady state) is determined by the motion and must be solved for as part of the problem. Indeed, the analogy should carry over to flow at arbitrary Reynolds number and similar equations will apply; it is only the constitutive relations that will distinguish a viscous suspension from a bubbly liquid.

The only remaining quantity to model is the heat flux vector $\langle\boldsymbol{q}\rangle$. In homogeneous flow this term is not important, but in inhomogeneous flows it plays a very important role in transporting fluctuational motion to regions of low shear rate and high particle concentration. A simple argument suggests a Fourier law:

$$
\langle\boldsymbol{q}\rangle=-\left\langle\boldsymbol{\Sigma}^{\prime} \cdot \boldsymbol{u}^{\prime}\right\rangle \sim-\eta \eta_{p}\left\langle\nabla \boldsymbol{u}^{\prime} \cdot \boldsymbol{u}^{\prime}\right\rangle \sim-\eta \kappa(\phi) \nabla T,
$$

where $\kappa(\phi)$, the 'thermal conductivity', should be proportional to the particle viscosity.

To see how the temperature introduces quadratic non-local effects, consider again the fully developed flow in the $x$-direction with all variations in the $y$-direction occurring on the macroscopic lengthscale $H$. The energy balance becomes

$$
\eta_{p}(\phi)\left(\frac{\mathrm{d}\langle u\rangle}{\mathrm{d} y}\right)^{2}-\alpha(\phi) T+\epsilon^{2} \frac{\mathrm{d}}{\mathrm{d} y}\left(\kappa(\phi) \frac{\mathrm{d} T}{\mathrm{~d} y}\right)=0,
$$

where $\epsilon=a / H$. Here, the temperature has been non-dimensionalized by $(a \dot{\gamma})^{2}$. When $\epsilon$ is small we have the simple algebraic balance

$$
T=\frac{\eta_{p}}{\alpha}\left(\frac{\mathrm{d}\langle u\rangle}{\mathrm{d} y}\right)^{2},
$$

which restates that fact that the temperature scales as the shear rate squared in homogeneous shear flow. If we now solve for $T$ by successive substitution from (57) into (56), and use this in (50) for the pressure, the desired quadratic non-local terms are produced. Thus, the introduction of the suspension temperature and its balance equation (54) give the desired non-local description for the stress.

Furthermore, we can see that $\epsilon^{2}$ multiplies the highest derivative in (56) and therefore whenever the shear rate vanishes there will be boundary layers whose thickness scales as $\epsilon$. Thus, the key difference between the two models is that the suspension balance model is non-local and takes account of the flux of fluctuational motion, while the diffusive flux model does not. This flux is important in two regions. Where the shear rate is zero, the conduction of fluctuational motion will result in a finite temperature, removing the cusp in the density profile that the diffusive model predicts (cf. figure 14a). Though the small parameter $\epsilon^{2}$ multiplies the conduction term in (56), the conductivity $\kappa(\phi)$ diverges as $\phi$ approaches maximum packing, thus ensuring that the conduction of fluctuational energy is important. The conduction of fluctuational motion is also important in the boundary layers near the walls as there is a rapid variation in $T$ within a layer of thickness $\epsilon$, which is precisely as seen in the simulations (cf. figure 7). 
To fully close the set of governing equations, functional forms for $\eta_{p}(\phi), p(\phi), \alpha(\phi)$, $c(\phi), \kappa(\phi)$, and $\boldsymbol{A}(\phi)$ have to be specified. While these functions can be measured from independent experiments or simulations, and moreover from homogeneous shear experiments (with the possible exception of $\kappa(\phi)$ ), we do not attempt to do so here and have instead chosen reasonable forms that reflect their qualitative variation with $\phi$. The particle viscosity can be derived from the extensive data that is available for the suspension viscosity, $\eta_{s}$. At small $\phi, T$ should be proportional to $\phi$ and $\Pi$ to $\phi^{2}$, since at least two particle interactions are needed to give rise to the temperature and pressure. And since $p(\phi)$ must diverge as $\phi \rightarrow \phi_{m}$, since its origin is similar to that of the viscosity, we choose

and

$$
\begin{gathered}
p(\phi)=\phi^{1 / 2}\left[\left(1-\phi / \phi_{m}\right)^{-2}-1\right], \\
\alpha(\phi)=k_{\alpha} \eta_{0}(\phi) / \phi,
\end{gathered}
$$

the latter reflecting the fact that even at close packing the temperature remains finite. Similarly, the conductivity $\kappa$ is assumed to be of the form

$$
\kappa=k_{\kappa} \eta_{p},
$$

where $k_{\alpha}$ and $k_{\kappa}$ are constants. Since we consider primarily steady flows, we shall not need $c(\phi)$. We shall also only investigate rectilinear flows here and will not need the normal-stress-difference functions.

We now have a closed set of equations by which, with appropriate boundary conditions, we can describe suspension transport. In future work we shall attempt to determine the phenomenological functions by Stokesian Dynamics simulation and by experiment.

To illustrate the behaviour and predictions of this suspension balance model, we shall discuss the case of pressure-driven flow in a channel. In wide-gap Couette flow there is no region of zero shear rate and the suspension balance model reduces to the diffusive flux model (apart from wall boundary layers of $O(E)$ ), which is in good agreement with experiment (Phillips et al. 1992). For pressure-driven flow in a channel of width $H$, the characteristic velocity scale is $\langle u\rangle=G H^{2} / \eta$, where $G$ is the magnitude of the pressure gradient, the temperature is scaled with $\langle u\rangle^{2}(a / H)^{2}$ and the distance with $H$. In the suspension balance model the dimensionless governing equations for steady fully developed flow are

$$
\frac{\mathrm{d}}{\mathrm{d} y}\left(\eta_{p}(\phi) \frac{\mathrm{d}\langle u\rangle_{p}}{\mathrm{~d} y}\right)+1-\epsilon^{-2} \frac{9}{2} \phi f^{-1}\left(\langle u\rangle_{p}-\langle u\rangle\right)=0,
$$

and

$$
\frac{\mathrm{d}}{\mathrm{d} y}\left(p(\phi) T^{1 / 2}\right)=0,
$$

where $u$ denotes the $x$-component of velocity. The energy balance is the same as (56).

From (58), it is immediately clear that to achieve a balance, $\langle u\rangle_{p}=\langle u\rangle+O\left(\epsilon^{2}\right)$, i.e. there is no phase slip between the particles and the fluid. This is an important and immediate conclusion from the particle balance and is borne out in our simulations (cf. table 1). Note that the diffusive flux model assumes that there is no phase slip, but with the suspension balance model we predict this result. Since there is no phase slip, the momentum balance for the entire suspension replaces (58):

$$
\frac{\mathrm{d}}{\mathrm{d} y}\left(\eta_{s}(\phi) \frac{\mathrm{d}\langle u\rangle}{\mathrm{d} y}\right)=-1 .
$$


Equations (56), (59), and (60) form a set of ordinary differential equations for the field variables $u, T$ and $\phi$. The $y$-momentum balance (59) simply states that the pressure across the channel remains constant and serves as the equation to determine $\phi$.

To solve the above set of differential equations, appropriate boundary conditions must be chosen at the centre and the walls of the channel. At the channel centre, symmetry requires that

$$
\frac{\mathrm{d}\langle u\rangle}{\mathrm{d} y}=\frac{\mathrm{d} T}{\mathrm{~d} y}=0 \quad \text { at } \quad y=0 .
$$

Note that though we expect $\mathrm{d} \phi / \mathrm{d} y=0$ at the centre, this need not be specified as it is automatically ensured by the $y$-momentum balance. At the walls, the no-slip condition is used for the particle velocity and the results of the simulations suggest that the temperature vanishes as well,

$$
u=T=0 \quad \text { at } \quad y=1 .
$$

With the above boundary conditions and the constraint on the average particle concentration

$$
\int_{0}^{1} \phi \mathrm{d} y=\phi^{b},
$$

(56), (59), and (60) can now be solved for the $\phi, u$ and $T$. Because of the small parameter $\epsilon^{2}$ we can expect to see boundary layers in the temperature (and concentration) fields. The derivatives of the flow variables are large within the boundary layers and the governing equations are therefore stiff.

The governing equations of the suspension temperature model for channel flow were solved by using a variable-step stiff ODE solver. The coefficients for $\alpha$ and $\kappa$ are

$$
k_{\alpha}=0.19 \text { and } k_{\kappa}=0.17 \text {. }
$$

Note that there is no need for a coefficient in the pressure function because only the ratio of $p$ to $\alpha$ matters. The numerical factors for $\alpha$ and $\kappa$ were chosen so as to achieve a reasonable fit with the simulation results for $\phi_{A}^{b}=0.45$ and $H / a=18.32$. In order to compare the results of the models with the simulations, $\phi_{m}$ is taken to be equal to 0.83 , the maximum-packing areal fraction in a monolayer (Kausch, Fesko \& Tschoegl 1971).

Figure 14 shows a comparison of the predictions of the two models with the simulation results for a channel width of 18.32 and bulk areal fraction of 0.45 . The contrast between the model of Phillips et al. (1992) and ours is apparent - their model predicts a cusp in the density profile with $\phi=\phi_{m}$ at the centre. The density profile predicted by our model, on the other hand, is almost identical to that observed in the simulation - the slope at the centre is zero (as expected from symmetry) and the concentration is well below the maximum-packing value. This important difference is because the theory of Phillips et al. is 'local' in the sense that the diffusivity is proportional to the local shear rate, while our theory is non-local owing to the presence of the energy flux. This allows for a non-zero temperature at the channel centre and hence a finite particle diffusivity. The velocity profiles predicted by the two models and the simulation are in much better agreement, confirming that the average particle velocities are relatively insensitive to the concentration distribution in the channel. The temperatures predicted by our model are in good agreement with those observed in the simulation except near the channel centre, where the theory under predicts the temperature. We should point out, however, that no attempt was made to 'fit' the theory to the simulations by choosing appropriate forms for the dimensionless 

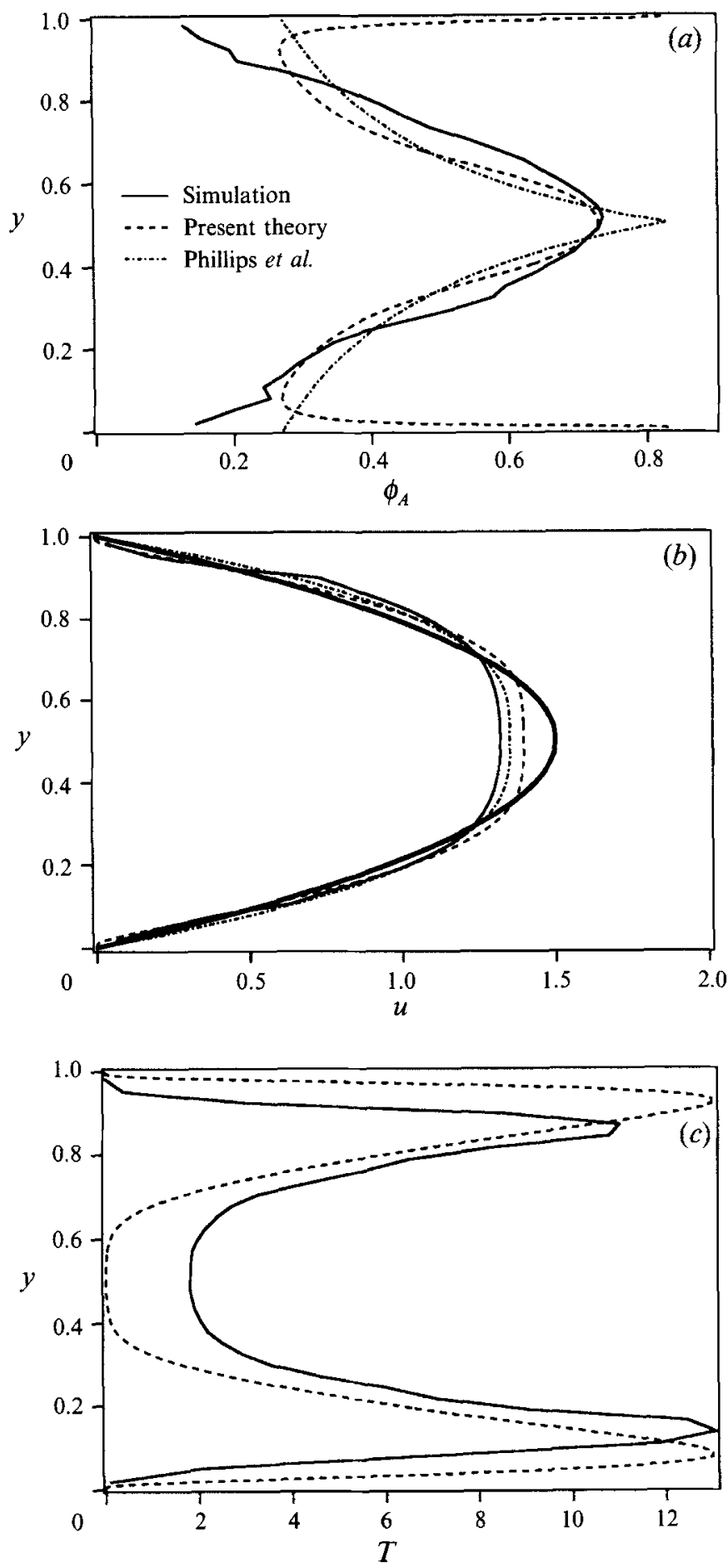

FIGURE 14. Comparison of the concentration $(a)$, velocity $(b)$ and temperature $(c)$ fields predicted by the diffusive flux and the suspension balance models for $H / a=18.32$ and $\phi_{A}^{b}=0.45$ with the data from simulation $J$. The dark line in $(b)$ is the parabolic velocity profile for a Newtonian fluid. 

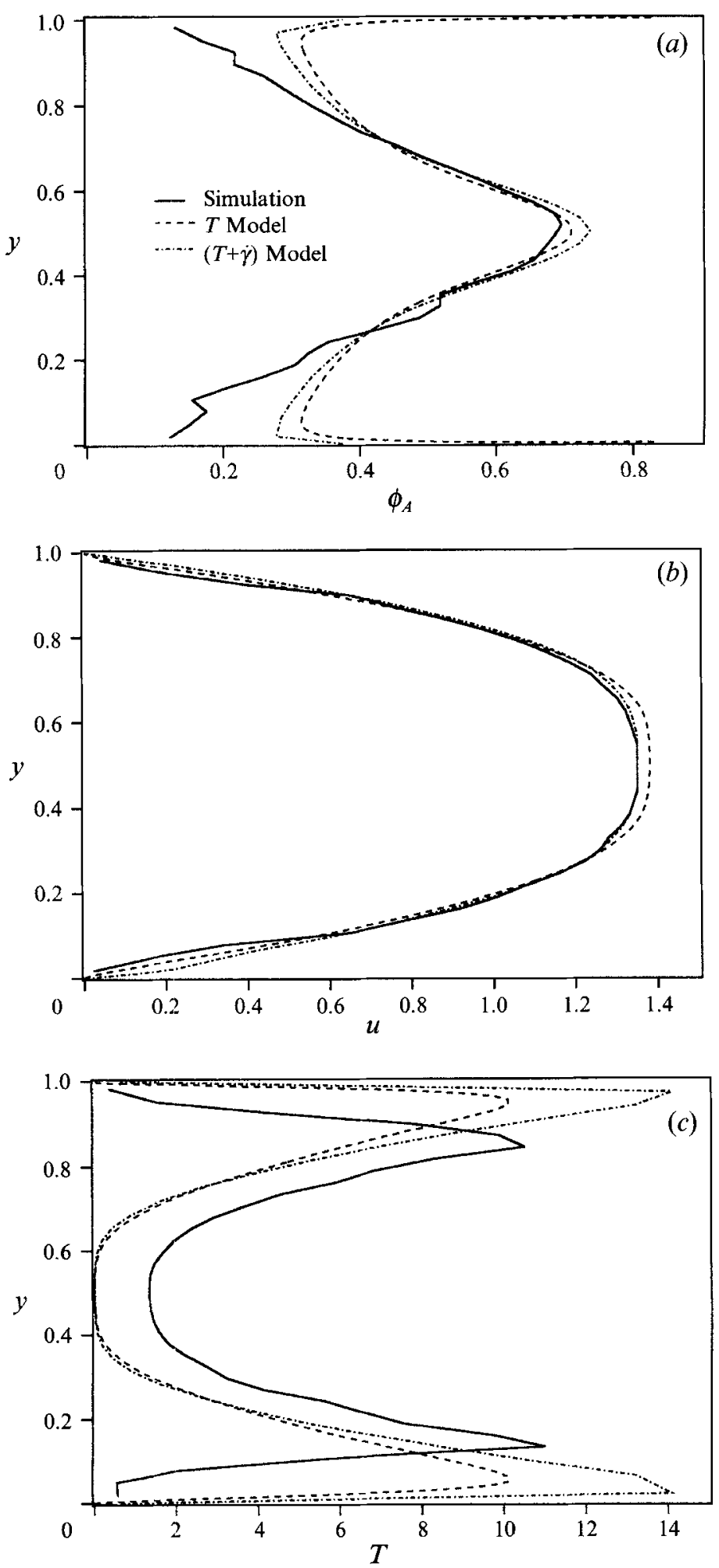

FIGURE 15. Comparison of the concentration $(a)$, velocity $(b)$ and temperature $(c)$ predicted by the two pressure models, (50) and (61), for $\phi^{b}=0.45$ and $H / a=18.32$. 
functions $p, \alpha$ and $\kappa$. It is our intention to determine the forms of these functions by independent means (e.g. from simulations of homogeneous shear flow) and then use them to predict the results for more complicated flows (such as tube flow, wide-gap Couette flow, etc.).

The concentration profiles predicted by the suspension balance model display a sharp upturn to maximum packing in the $O(\epsilon)$ thin boundary layers adjacent to the walls, which results from setting the temperature to be zero at the walls. This upturn is not seen in the simulations and thus the model requires modification to remedy this fact. One possibility is to model the pressure as a combination of fluctuational and systematic terms, i.e. simply combining (39) and (50) into a single expression:

$$
\Pi=\frac{1}{2}\left(p(\phi) T^{1 / 2}+\bar{p}(\phi) \dot{\gamma}\right) .
$$

In homogeneous flow all forms for the pressure are equivalent. But with (61) near the centreline where $\dot{\gamma}$ vanishes the pressure will be set by $T^{1 / 2}$, while near the walls where $T$ is zero, it will be set by $\dot{\gamma}$. Figure 15 compares the results of (61) for the pressure with the pure temperature model. As expected the concentration upturn near the walls is dramatically reduced; the amount of upturn can be adjusted by choosing a different ratio of $T^{1 / 2}$ to $\dot{\gamma}$.

It is also possible to lessen the upturn in the concentration near the walls by modifying the boundary condition on the temperature. The simulation results suggest that $T=0$, but a local energy balance of the type used in granular flow (Jenkins \& McTigue 1990) might be appropriate and lead to a small but finite temperature at the wall, which will reduce the concentration there. Clearly, more work needs to be done in modelling the boundary conditions at the wall and in examining whether a systematic term should be added to the pressure.

\section{Conclusions}

Stokesian Dynamics simulations of pressure-driven flow in a channel were conducted for a suspension of monodisperse spherical particles. The simulations were performed for a range of the ratio $H / a$ (where $H$ is the width of the channel and $a$ is the particle radius) and the bulk particle concentration $\phi_{A}^{b}$. Starting from a homogeneous dispersion of particles in the channel, the simulations reveal that particles gradually migrate towards the centre of the channel, resulting in a considerably inhomogeneous distribution and blunting, or flattening, of the particle velocity profile at steady state. Recent experimental studies of pressure-driven flow in a tube or channel have reported particle migration and velocity blunting, but there are significant disparities in their findings on the degree and extent of particle migration. While errors in the measurement techniques are, no doubt, responsible for some of the differences in the observations, an important factor is the transition length of the channel (or tube) that is required to achieve steady state. We have shown that this transition length scales as $H^{3} / a^{2}$, which can be much greater than the development length for the parabolic velocity profile for a homogeneous Newtonian fluid. Most of the measurements in earlier studies were made within the transition length, perhaps accounting for some of the disparities in their findings.

In addition to the concentration and velocity profiles, the particle temperature profile has also been reported for the simulations in this work. The temperature is a measure of the velocity fluctuations of the particles about their local mean and is of interest because it is closely related to the diffusivity and because spatial variations in temperature can be used to develop a non-local theory of suspension transport, which 
is necessary to explain the experimental observations. The temperature is lowest at the channel centre and increases until it reaches a maximum at a distance of roughly two particle diameters from the wall, beyond which point it decreases and vanishes at the walls.

Migration of particles towards the channel centre occurs even without the presence of any non-hydrodynamics force between the particles. However, the stronglubrication interactions between particles result in the formation of persistent clusters among particles which impede their motion and hence limit the extent of particle migration. With the inclusion of a short-range repulsive force between the particles, these clusters are broken and the particle concentration at the centre is greater than when no inter-particle force is present. However, the behaviour remains unchanged over three decades of the range (or strength) of the inter-particle force, showing that it has no effect other than breaking the strong-lubrication interactions.

The effect of increasing the channel width is a slight rise in the particle concentration and a fall in the velocity at the centre. The temperature, however, is strongly influenced by the channel width and decreases everywhere but in the layer adjacent to the walls. The thickness of this layer (scaled with $H$ ), within which the temperature falls from its maximum to zero at the wall, decreases monotonically with increasing $H / a$. The effect of the bulk particle concentration is as expected - with increasing $\phi_{A}^{b}$, the particle concentration throughout the channel increases and so do the degree of velocity blunting and the particle temperature.

The model for shear-induced diffusion proposed by Leighton \& Acrivos assumes that the diffusivity of particles is proportional to the local rate of strain in the suspension. Though this is indeed the case in homogeneous shear flow, it is not necessarily valid in general - the diffusivity is properly thought of as proportional to the square root of the particle temperature. Thus, the particle temperature is a fundamental quantity in any macroscopic description of suspension flow and a conservation equation for the fluctuational motion is needed to complete the description. We have proposed a model where there is no diffusive motion of particles, which are considered as a material phase. Migration occurs through the requirement that the macroscopic pressure be constant perpendicular to the direction of mean motion. Further, the presence of normal stress differences allows the possibility to prevent migration in torsional flow, which is in agreement with recent experiments. (Note that for wide-gap Couette flow the normal stress differences do not prevent migration.) Finally, linking the suspension pressure to the fluctuational motion of the particles, as described by the suspension temperature and the balance law for fluctuational energy, introduces a non-locality into the description of suspension behaviour and removes the difficulties in regions of zero shear rate.

The model developed in this work can also be applied to other flow geometries where particle migration is of importance, such as in wide-gap Couette flow and viscous resuspension, and we expect to do so in future studies. The model can also be applied to unsteady flows, and since it contains the diffusive flux model of Phillips et al. (1992) as a special case, the temporal behaviour will be of the same diffusive-like character.

Given that particles migrate in inhomogeneous flows, it is important to investigate the stability of a homogeneous flow. For simple shear flow with velocity in the $x$ direction and gradient in the $y$-direction, the linear stability analysis leads directly to (47) for the concentration disturbance. The suspension momentum balance requires that

$$
\frac{\partial \dot{\gamma}}{\partial y}=-\dot{\gamma} \frac{\eta_{s}^{\prime}}{\eta_{s}} \frac{\partial \phi}{\partial y},
$$


which, when combined with (47), leads to the stability requirement

$$
\eta_{s} f \frac{\mathrm{d}}{\mathrm{d} \phi}\left(\frac{\bar{p}}{\eta_{s}}\right)>0 .
$$

Since both $\eta_{s}$ and $f$ are everywhere positive functions, the stability is governed by the variation of $\bar{p} / \eta_{s}$ with $\phi$. This competition between the pressure function and the viscosity arises as follows. When a fluctuation increases the concentration, the pressure is increased, which then acts to expel particles from the region of increased concentration. The opposite occurs for fluctuations that decrease the concentration. This action is to stabilize the suspension. On the other hand, when the concentration is increased, the viscosity increases and the shear rate must decrease so that the stress remains constant. With a lower shear rate, particles will tend to migrate to this region, further enhancing the increased concentration. The opposite occurs where the concentration decreases. This action is to destabilize the system. Stability is then governed by whether the pressure increase due to concentration outweighs the decrease due to the change in shear rate. Little is known about the pressure function in viscous suspensions, but it seems reasonable that $\bar{p}$ will increase as rapidly as $\eta_{s}$ as maximum packing is approached, and therefore homogeneous suspension flow should be stable. (Note that the constitutive equation we have used for the pressure ensures stability.) There have been no reports of instabilities in homogeneous shear flow, but clearly this is an interesting and important problem to study.

Although there is no net diffusion in the suspension balance model, it is easy to show that the model can be used to derive a diffusion equation for tracer particles in shear flow, i.e. the diffusion equation describing the motion of a small fraction of the particles which are marked so as to differentiate them from the rest. A mass and momentum balance applied to the small fraction of tracer particles, which now constitute a new phase distinguished from the majority of the particles, shows that the driving force for their motion relative to the mean is the gradient in the "partial pressure' of the tracer particles and results in

$$
D=\frac{2}{9} \dot{\gamma} a^{2} f(\phi) \bar{p}(\phi)=\frac{2}{9} a f(\phi) p(\phi) T^{1 / 2} .
$$

Independent measurements of $D, T, f, \bar{p}$ and $p$ in homogeneous flow can be used to check this model prediction.

It should also be appreciated that the phenomenological coefficients introduced in the suspension balance model can all be obtained in independent experiments in homogeneous flow. Suspension viscosities, and to a limited extent normal stresses, have been measured for many years, but the suspension pressure has not. Clearly experiments to measure the suspension pressure and temperature are needed to fully understand and explain suspension behaviour.

The present model can also be extended to more general situations, such as when Brownian motion is important (i.e. finite Péclet number) or when the particles exert electrostatic or other non-hydrodynamic forces on each other. In these situations, the constitutive relations for the stress and energy dissipation require modification, but the structure of the governing equations remains the same. Indeed, the ideas underlying this approach are also applicable when inertial effects are important, such as in fluidized beds or in the flow of bubbly liquids, and may form the basis for the rational modelling of multiphase flows.

This work was supported by the Office of Naval Research under Grant No. N0001490-J-1945. The authors also wish to acknowledge the San Diego Supercomputer Center 
for providing computational facilities. Discussions with Mr J. F. Morris regarding the origin of velocity fluctuations in low-Reynolds-numbers flows were especially helpful.

\section{REFERENCES}

Abbott, J. R., Tetlow, N., Graham, A. L., Altobelli, S. A., Fukushima, E., Mondy, L. A. \& STEPHENS, T.S. 1991 Experimental observations of particle migration in concentrated suspensions: Couette flow. J. Rheol. 35, 773.

BATCHELOR, G. K. 1970 The stress in a suspension of force-free particles. J. Fluid Mech. 41, 545.

Bird, R. B., Armstrong, R. C. \& Hassager, O. 1977 Dynamics of Polymeric Liquids. Wiley.

BonNeCAZE, R. T. \& BRADY, J. F. 1992 Yield stresses in electrorheological fluids. J. Rheol. 36, 73.

Bossis, G. \& BRADY, J. F. 1987 Self-diffusion of Brownian particles in concentrated suspensions under shear. J. Chem. Phys. 87, 5437.

Brady, J. F. 1993 a Brownian motion, hydrodynamics and the osmotic pressure. J. Chem. Phys. 98, 3335.

BRady, J. F. $1993 b$ The rheological behavior of concentrated colloidal dispersions. J. Chem. Phys. $99,567$.

BRADY, J. F. \& Bossis, G. 1985 The rheology of concentrated suspensions of spheres in simple shear flow by numerical simulation. J. Fluid Mech. 155, 105.

Brady, J. F. \& Bossis, G. 1988 Stokesian Dynamics. Ann. Rev. Fluid Mech. $20,111$.

Brady, J. F., Phillips, R. J., Lester, J. C. \& Bossis, G. 1988 Dynamic simulation of hydrodynamically interacting suspensions. J. Fluid Mech. 195, 257.

Chow, A. W., Sinton, S. W., Iwamiya, J. H. \& StePhens, T. S. 1994 Shear-induced migration in Couette and parallel-plate viscometers: NMR imaging and stress measurements. Phys. Fluids (submitted).

Drew, D. A. \& LAHEY, R. T. 1993 Analytical modeling of multiphase flow. In Particular Two-Phase Flow (ed. M. Roco), p. 509. Butterworths.

DuRlofsKY, L. J. \& BRADY, J.F. 1989 Dynamic simulation of bounded suspensions of hydrodynamically interacting particles. J. Fluid Mech. 200, 39.

Eckstein, E. C., Bailey, D. G. \& Shapiro, A. H. 1977 Self-diffusion of particles in shear flow of a suspension. J. Fluid Mech. 79, 191.

Gadala-Maria, F. \& ACrivos, A. 1980 Shear induced structure in a concentrated suspension of solid spheres. $J$. Rheol. 24, 799.

HookHAM, P. 1986 Concentration and velocity measurements in suspensions flowing through a rectangular channel. Ph.D. thesis, California Institute of Technology.

IRVING, J. H. \& KIRKWOOD, J. G. 1950 The statistical mechanical theory of transport processes. IV. The equations of hydrodynamics. J. Chem. Phys. 18 (6), 817.

JefFreY, D. J., MoRris, J. F. \& BRADY, J. F. 1993 The pressure moments for two rigid spheres in low Reynolds number flow. Phys. Fluids A 5, 2317.

Jenkins, J. T. \& MCTIGUE, D. F. 1990 Transport processes in concentrated suspensions: the role of particle fluctuations. In Two Phase Flows and Waves (ed. D. D. Joseph \& D. G. Schaeffer). Springer.

Karnis, A., Goldsmith, H. L. \& Mason, S. G. 1966 The kinetics of flowing dispersions: Concentrated suspensions of rigid particles. J. Colloid Interface Sci. 22, 531.

Kausch, H. H., Fesko, D. G. \& Tschoegl, N. W. 1971 The random packing of circles in a plane. J. Colloid Interface Sci. 37, 603.

KoH, C. J. 1991 Experimental and theoretical studies on two-phase flows. Ph.D thesis, California Institute of Technology.

Кон, C. J., Ноокнам, P. \& Leal, L. G. 1994 An experimental investigation of concentrated suspension flows in a rectangular channel. J. Fluid Mech. 266, 1.

Leighton, D. \& ACRIvos, A. 1987 a Measurement of self-diffusion in concentrated suspension of spheres. J. Fluid Mech. 177, 109.

Leighton, D. \& ACrivos, A. $1987 b$ The shear-induced migration of particles in concentrated suspensions. J. Fluid Mech. 181, 415. 
Phan, S. E. \& Leighton, D. T. 1994 Measurement of the shear-induced tracer diffusivity in concentrated suspensions. J. Fluid Mech. (submitted).

Phillips, R. J., Armstrong, R. C., Brown, R. A., Graham, A. \& Abbott, J. R. 1992 A constitutive model for concentrated suspensions that accounts for shear-induced particle migration. Phys. Fluids A 4, 31.

Phillips, R. J., Brady, J. F. \& Bossis, G. 1988 Hydrodynamic transport properties of hard-sphere dispersions. I. Suspensions of freely mobile particles. Phys. Fluids 31, 3462.

Phung, T. 1993 Behavior of concentrated colloidal suspensions by Stokesian Dynamics simulation. $\mathrm{Ph} . \mathrm{D}$ thesis, California Institute of Technology.

Phung, T. \& Brady, J. F. 1992 Microstructural fluids: structure, diffusion and rheology of colloidal dispersions. In Slow Dynamics in Condensed Matter (ed. K. Kawasaki et al.). AIP Conf. Proc. vol. 256, p. 391.

Segré, G. \& Silberberg, A. 1962 Behaviour of macroscopic rigid particles in Poiseuille flow. J. Fluid Mech. 14, 115.

Sinton, S. W. \& Chow, A. W. 1991 NMR flow imaging of fluids and solid suspensions in Poiseuille flow. J. Rheol. 35, 735. 